\title{
Poisson process Fock space representation, chaos expansion and covariance inequalities
}

\author{
Günter Last* and Mathew D. Penrose $\dagger$
}

September 14, 2009

\begin{abstract}
We consider a Poisson process $\eta$ on an arbitrary measurable space with an arbitrary sigma-finite intensity measure. We establish an explicit Fock space representation of square integrable functions of $\eta$. As a consequence we identify explicitly, in terms of iterated difference operators, the integrands in the Wiener-Itô chaos expansion. We apply these results to extend well-known variance inequalities for homogeneous Poisson processes on the line to the general Poisson case. The Poincaré inequality is a special case. Further applications are covariance identities for Poisson processes on (strictly) ordered spaces and Harris-FKG-inequalities for montone functions of $\eta$.
\end{abstract}

Key words and phrases. Poisson process, chaos expansion, derivative operator, KabanovSkorohod integral, Malliavin calculus, Poincaré inequality, variance inequalities, infinitely divisible random measure

\section{Introduction}

The aim of this paper is to develop and to exploit the basic Fock space structure of a Poisson process $\eta$ on a measurable space $(\mathbb{Y}, \mathcal{Y})$ with $\sigma$-finite intensity measure $\lambda$. In contrast to the literature we do not make any restrictions of generality, neither imposing a topological structure on the phase space $(\mathbb{Y}, \mathcal{Y})$, nor assuming the measure $\lambda$ to be continuous. Moreover, our results are more explicit than what was available previously. We use a probabilistic and non-technical approach that is based on only a few basic properties of a Poisson process.

We now describe the contents of this paper in more detail. The underlying probability space is denoted by $(\Omega, \mathcal{F}, \mathbb{P})$. We interpret the Poisson process $\eta$ as a random element in the space $\mathbf{N}:=\mathbf{N}(\mathbb{Y})$ of integer-valued $\sigma$-finite measures $\mu$ on $\mathbb{Y}$ equipped with the

\footnotetext{
*Institut für Stochastik, Universität Karlsruhe (TH), 76128 Karlsruhe, Germany. Email: last@math.uni-karlsruhe.de

${ }^{\dagger}$ Department of Mathematical Sciences, University of Bath, Bath BA2 7AY, United Kingdom, Email: m.d.penrose@bath.ac.uk

${ }^{\ddagger}$ Partially supported by the Alexander von Humboldt Foundation through a Friedrich Wilhelm Bessel Research Award.
} 
smallest $\sigma$-field $\mathcal{N}$ making the mappings $\mu \mapsto \mu(B)$ measurable for all $B \in \mathcal{Y}$. For $y \in \mathbb{Y}$ the difference operator $D_{y}$ (also known as the add one cost operator) is given as follows. For any measurable $f: \mathbf{N} \rightarrow \mathbb{R}$ the function $D_{y} f$ on $\mathbf{N}$ is defined by

$$
D_{y} f(\mu):=f\left(\mu+\delta_{y}\right)-f(\mu), \quad \mu \in \mathbf{N},
$$

where $\delta_{y}$ is the Dirac measure located at a point $y \in \mathbb{Y}$. Iterating this definition, for $n \geq 2$ and $\left(y_{1}, \ldots, y_{n}\right) \in \mathbb{Y}^{n}$ we define a function $D_{y_{1}, \ldots, y_{n}}^{n} f: \mathbf{N}(\mathbb{Y}) \rightarrow \mathbb{R}$ inductively by

$$
D_{y_{1}, \ldots, y_{n}}^{n} f:=D_{y_{1}}^{1} D_{y_{2}, \ldots, y_{n}}^{n-1} f
$$

where $D^{1}:=D$ and $D^{0} f=f$. As we shall see, the operator $D_{y_{1}, \ldots, y_{n}}^{n}$ is symmetric in $y_{1}, \ldots, y_{n}$. We define symmetric and (as it turns out) measurable functions $T_{n} f$ on $\mathbb{Y}^{n}$ by

$$
T_{n} f\left(y_{1}, \ldots, y_{n}\right):=\mathbb{E} D_{y_{1}, \ldots, y_{n}}^{n} f(\eta),
$$

and we set $T_{0} f:=\mathbb{E} f(\eta)$, whenever these expectations are defined.

By $\langle\cdot, \cdot\rangle_{n}$ we denote the scalar product in $L^{2}\left(\lambda^{n}\right)$ and by $\|\cdot\|_{n}$ the associated norm. Let $\mathbb{P}_{\eta}$ denote the distribution of $\eta$. Then $L^{2}\left(\mathbb{P}_{\eta}\right)$ is the space of all measurable $f: \mathbf{N} \rightarrow \mathbb{R}$ satisfying $\mathbb{E} f(\eta)^{2}<\infty$. For $n \in \mathbb{N}$ let $\mathbf{H}_{n}$ be the space of symmetric functions in $L^{2}\left(\lambda^{n}\right)$, and let $\mathbf{H}_{0}:=\mathbb{R}$. Our first result says that the mapping $f \mapsto\left(T_{n}(f)\right)_{n \geq 0}$ is an isometry from $L^{2}\left(\mathbb{P}_{\eta}\right)$ to the Fock space given by the direct sum of the spaces $\mathbf{H}_{n}, n \geq 0$, with $L^{2}$ norms scaled by $n !^{-1 / 2}$, as we describe in more detail in Section 2 .

Theorem 1.1. Let $f \in L^{2}\left(\mathbb{P}_{\eta}\right)$. Then

$$
\mathbb{E} f(\eta)^{2}=(\mathbb{E} f(\eta))^{2}+\sum_{n=1}^{\infty} \frac{1}{n !}\left\|T_{n} f\right\|_{n}^{2} .
$$

If also $g \in L^{2}\left(\mathbb{P}_{\eta}\right)$, then more generally,

$$
\mathbb{E} f(\eta) g(\eta)=(\mathbb{E} f(\eta))(\mathbb{E} g(\eta))+\sum_{n=1}^{\infty} \frac{1}{n !}\left\langle T_{n} f, T_{n} g\right\rangle_{n} .
$$

We shall use Thoerem 1.1 to provide a new proof of the following family of inequalities for the variance of Poisson functionals, which were previously given by Houdré and PerezAbreu [12] (but with proof only for a Gaussian analogue) and by Privault [38] for the case of normal martingales (including the homogeneous Poisson process on the line). These estimates involve alternating sums with similar terms to those in (1.4), except that we take the expectation outside the inner product. Accordingly, let $D^{n} f(\eta)$ denote the mapping $\left(y_{1}, \ldots, y_{n}\right) \mapsto D_{y_{1}, \ldots, y_{n}}^{n} f(\eta)$.

Theorem 1.2. Let $f \in L^{2}\left(\mathbb{P}_{\eta}\right)$ and $k \in \mathbb{N}$ be such that

$$
\mathbb{E}\left\|D^{n} f(\eta)\right\|_{n}^{2}<\infty, \quad n=1, \ldots, 2 k .
$$

Then

$$
\sum_{n=1}^{2 k} \frac{(-1)^{n+1}}{n !} \mathbb{E}\left\|D^{n} f(\eta)\right\|_{n}^{2} \leq \operatorname{Var}[f(\eta)] \leq \sum_{n=1}^{2 k-1} \frac{(-1)^{n+1}}{n !} \mathbb{E}\left\|D^{n} f(\eta)\right\|_{n}^{2} .
$$

The first inequality of (1.7) is an equality if and only if $D_{y_{1}, \ldots, y_{2 k+1}}^{2 k+1} f(\eta)=0$ almost surely, for $\lambda^{2 k+1}$-almost all $\left(y_{1}, \ldots, y_{2 k+1}\right) \in \mathbb{Y}^{2 k+1}$. The second inequality of $(1.7)$ is an equality if and only if $D_{y_{1}, \ldots, y_{2 k}}^{2 k} f(\eta)=0$ almost surely, for $\lambda^{2 k}$-almost all $\left(y_{1}, \ldots, y_{2 k}\right) \in \mathbb{Y}^{2 k}$. 
The case $k=1$ of the right hand inequality in (1.7) says that

$$
\operatorname{Var} f(\eta) \leq \mathbb{E} \int\left(f\left(\eta+\delta_{y}\right)-f(\eta)\right)^{2} \lambda(d y),
$$

and is known as the Poincaré inequality for the variance of Poisson functionals. In the present generality (1.8) was previously derived by [43] using an explicit martingale representation. The same method was used earlier in [5] to establish the result for infinitely divisible random vectors with independent components. Again our proof is different.

In our opinion the Poincaré inequality is a fundamental property of Poisson processes; for an example of its application see [10]. It is related to the well-known Efron-Stein inequality [8] for the variance of a symmetric function of $n$ independent $\mathbb{Y}$-valued random variables.

In Section 3 we consider for $n \in \mathbb{N}$ the multiple Wiener-Itô integral $I_{n}(g)$ of a symmetric function $g \in L^{2}\left(\lambda^{n}\right)$ with respect to the compensated Poisson process $\hat{\eta}$ (see $[41,16,30]$ ). In fact we shall follow Liebscher [22] in defining this integral in the general case, that is without assuming that $\lambda$ is continuous as in $[16,30]$. For $c \in \mathbb{R}$ we set $I_{0}(c):=c$. Itô's [16] and Wiener's [41] famous chaos expansion of square integrable random variables says that every function $f \in L^{2}\left(\mathbb{P}_{\eta}\right)$ can be decomposed uniquely as a sum of variables of the form $I_{n}\left(f_{n}\right)$ with $\left(f_{n}\right)_{n \geq 0}$ in our Fock space. The following result identifies each function $f_{n}$ as $1 / n$ ! times the image of $f$ under the mean iterated difference operator $T_{n}$.

Theorem 1.3. Let $f \in L^{2}\left(\mathbb{P}_{\eta}\right)$. Then $T_{n} f \in L^{2}\left(\lambda^{n}\right), n \in \mathbb{N}$, and

$$
f(\eta)=\sum_{n=0}^{\infty} \frac{1}{n !} I_{n}\left(T_{n} f\right),
$$

where the series converges in $L^{2}(\mathbb{P})$. Moreover, if $g_{n} \in \mathbf{H}_{n}$ for $n \in \mathbb{N}_{0}$ satisfy $f(\eta)=$ $\sum_{n=0}^{\infty} \frac{1}{n !} I_{n}\left(g_{n}\right)$ with convergence in $L^{2}(\mathbb{P})$, then $g_{0}=\mathbb{E} f(\eta)$ and $g_{n}=T_{n} f, \lambda^{n}$-almost everywhere on $\mathbb{Y}^{n}$, for all $n \in \mathbb{N}$.

In the case $\mathbb{Y}=\mathbb{R}$, Theorem 1.3 has been obtained by Y. Ito ([17], see eqn (7.5) there). A less explicit version for the special case of Lévy processes (without Gaussian component) can be found as Theorem 4 in [23]. (The proof in [23] does not seem to justify its usage of iterated stochastic integrals, see e.g. Theorem 18.13 in [21] for the Brownian case.) Our proof is different from these, and applies to arbitrary $\sigma$-finite intensity measures. Theorem 1.3 and the isometry properties of stochastic integrals (see (3.5)) show that the isometry $f \mapsto\left(T_{n}(f)\right)_{n \geq 0}$ is in fact a bijection from $L^{2}\left(\mathbb{P}_{\eta}\right)$ onto the Fock space. They could also be used to deduce Theorem 1.1, but we shall proceed in the other direction, starting with Theorem 1.1 which is more fundamental. Neither its formulation nor its proof requires stochastic integration. For finite Poisson processes the operators $T_{n}$ had been previously used in $[28,29]$ to approximate the expectation of a Poisson functional, while [3] used (in a similar context) a closely related operator for more general point processes on the line.

Additional results in Section 3 are concerned with certain derivative and integral operators which are important in Malliavin calculus on Poisson spaces (see for example [32]). In Theorem 3.3, we provide a generalization to arbitrary $\sigma$-finite intensity measure (and new proof) of a result in [17] (see also [30]) identifying the difference operator with a stochastic linear derivative operator from $L^{2}\left(\mathbb{P}_{\eta}\right)$ to $L^{2}\left(\mathbb{P}_{\eta} \otimes \lambda\right)$. We also consider 
the stochastic Kabanov-Skorohod integral [11, 18, 19] which is a linear operator from $L^{2}\left(\mathbb{P}_{\eta} \times \lambda\right)$ to $L^{2}\left(\mathbb{P}_{\eta}\right)$ that is dual to the derivative operator. In Theorem 3.5 we provide a new proof of this duality for our more general setting, and a pathwise interpretation of the Kabanov-Skorohod integral, using a classical Campbell-type formula due to Mecke [25] for general Poisson processes.

In Section 5 we prove some results on covariances, including the following HarrisFKG inequality; see also [26], [4] (treating special Poisson processes) [43], and [9] for more general point processes. Given $B \in \mathcal{Y}$, a function $f: \mathbf{N}(\mathbb{Y}) \rightarrow \mathbb{R}$ is increasing on $B$ if $f\left(\mu+\delta_{y}\right) \geq f(\mu)$ for all $\mu \in \mathbf{N}(\mathbb{Y})$ and all $y \in B$. It is decreasing on $B$ if $(-f)$ is increasing on $B$. The formulation we give allows for functions which are increasing on some parts of $\mathbb{Y}$ and decreasing on others, which has occasionally been useful; see Lemma 14 on page 278 of [4], or page 878 of [34].

Theorem 1.4. Suppose $B \in \mathcal{Y}$. Let $f, g \in L^{2}\left(\mathbb{P}_{\eta}\right)$ be increasing on $B$ and decreasing on $\mathbb{Y} \backslash B$. Then

$$
\mathbb{E}[f(\eta) g(\eta)] \geq(\mathbb{E} f(\eta))(\mathbb{E} g(\eta)) .
$$

We shall derive Theorem 1.4 from the following result. For this result only, we make the extra assumption that $\mathbb{Y}$ is equipped with a transitive binary relation $<$ such that (i) $\{(y, z): y<z\}$ is a measurable subset of $\mathbb{Y}^{2}$ and (ii) for any $y, z \in \mathbb{Y}$ at most one of the relations $y<z$ and $z<y$ can be satisfied, and (iii) $<$ strictly orders the points of $\mathbb{Y}$ $\lambda$-a.e., that is

$$
\lambda(\mathbb{Y} \backslash\{z \in \mathbb{Y}: z<y \text { or } y<z\})=0, \quad y \in \mathbb{Y} .
$$

For any $\mu \in \mathbf{N}$ let $\mu_{y}$ denote the restriction of $\mu$ to $y_{\downarrow}:=\{z \in \mathbb{Y}: z<y\}$. Our final assumption on $<$ is that (iv) $(\mu, y) \mapsto \mu_{y}$ is a measurable mapping from $\mathbf{N} \times \mathbb{Y}$ to $\mathbf{N}$. In Section 5 we shall use Theorem 1.1 to derive the following identity for the covariance between two functions of $\eta$. The theorem requires a version of the conditional expectation $\mathbb{E}\left[D_{y} f(\eta) \mid \eta_{y}\right]$ that is jointly measurable in all arguments. Thanks to the independence properties of a Poisson process we can and will work with

$$
\mathbb{E}\left[D_{y} f(\eta) \mid \eta_{y}\right]:=\int D_{y} f\left(\eta_{y}+\mu\right) \Pi^{y}(d \mu)
$$

where $\Pi^{y}$ is the distribution of the restriction of $\eta$ to $\mathbb{Y} \backslash y_{\downarrow}$. By assumption (iv) and Fubini's theorem it follows that $\Pi^{y}(\cdot)$ is a kernel, that is $y \mapsto \Pi^{y}(A)$ is measurable for all measurable $A \subset \mathbf{N}$.

Theorem 1.5. Assume that $\mathbb{Y}$ is equipped with a transitive binary relation $<$ satisfying conditions (i)-(iv) above. For any $f \in L^{2}\left(\mathbb{P}_{\eta}\right)$,

$$
\mathbb{E} \int \mathbb{E}\left[D_{y} f(\eta) \mid \eta_{y}\right]^{2} \lambda(d y)<\infty
$$

and for any $f, g \in L^{2}\left(\mathbb{P}_{\eta}\right)$,

$$
\operatorname{Cov}[f(\eta), g(\eta)]=\mathbb{E} \int \mathbb{E}\left[D_{y} f(\eta) \mid \eta_{y}\right] \mathbb{E}\left[D_{y} g(\eta) \mid \eta_{y}\right] \lambda(d y)
$$


When $\lambda$ is the product of Lebesgue measure on $[0,1]$ and a $\sigma$-finite measure on some space $\mathbb{X}$, formula (1.14) is proved in [5] for special functions $f$, mentioned in [43] (under an additional assumption on $f$ ) and derived (in case of a finite and absolutely continuous intensity measure) in [35]. For normal martingales the result is stated in [14]. The version for infinitely divisible random vectors can be found in [5] and [13].

In Section 6 we shall discuss the Poincaré and Harris-FKG inequalities for infinitely divisible random measures. In Section 7 we describe some of the implications of our results in the case where $\mathbb{Y}$ is a finite set.

As has been discussed, some of our results already appear in the literature for special cases such as when the intensity measure is Lebesgue measure on $\mathbb{R}_{+}$. It may be possible to extend these existing results to the case where $\mathbb{Y}$ is a Borel space (a space that is Borel isomorphic to a Borel subset of $[0,1]$ ), by considering $\lambda$ as the image of Lebesgue measure under a measurable mapping from $\mathbb{R}_{+}$to $\mathbb{Y}$ (see Lemma 3.22 of [21]) and making an appropriate change of variables in the integrals. Nevertheless, we think our direct approach is worthwhile; as well as being applicable to an arbitrary intensity measure without any Borel condition, it provides a natural apporach which relies only on basic properties of Poisson processes, avoiding the technicalities of stochastic calculus seen in previous work.

\section{Fock space representation}

For any $n \in \mathbb{N}$ let $\mathbf{H}_{n}$ denote the space of all measurable functions $g: \mathbb{Y}^{n} \rightarrow \mathbb{R}$ that are square-integrable with respect to $\lambda^{n}$ and symmetric $\lambda_{n}$-a.e., equipped with the $L^{2}\left(\lambda^{n}\right)$ innner product $\langle\cdot, \cdot\rangle_{n}$ and corresponding norm norm $\|\cdot\|_{n}$ as in Section 1. Define $\mathbf{H}_{0}:=\mathbb{R}$. Consider the vector space $\mathbf{H}$ of all sequences $f=\left(f_{n}\right)_{n \geq 0}$ satisfying $f_{n} \in \mathbf{H}_{n}, n \geq 0$, and

$$
\sum_{n=0}^{\infty} \frac{1}{n !}\left\|f_{n}\right\|_{n}^{2}<\infty
$$

where $\left\|f_{0}\right\|_{0}:=\left|f_{0}\right|$. Equipped with the scalar product

$$
\langle f, g\rangle_{\mathbf{H}}:=\sum_{n=0}^{\infty} \frac{1}{n !}\left\langle f_{n}, g_{n}\right\rangle_{n}, \quad f=\left(f_{n}\right), g=\left(g_{n}\right) \in \mathbf{H}
$$

$\mathbf{H}$ becomes a Hilbert space; this is the Fock space that we consider in this paper. Meyer [27] gives an introduction into stochastic calculus on these spaces.

For any $f \in L^{2}\left(\mathbb{P}_{\eta}\right)$ we define $T f:=\left(T_{n} f\right)_{n \geq 0}$, where $T_{n} f$ is given at (1.3). Theorem 1.1 asserts that $T f \in \mathbf{H}$ for $f \in L^{2}\left(\mathbb{P}_{\eta}\right)$ and

$$
\mathbb{E} f(\eta) g(\eta)=\langle T f, T g\rangle_{\mathbf{H}}, \quad f, g \in L^{2}\left(\mathbb{P}_{\eta}\right)
$$

We prove these assertions in stages. Note first that

$$
D_{y_{1}, \ldots, y_{n}}^{n} f(\mu)=\sum_{J \subset\{1,2, \ldots, n\}}(-1)^{n-|J|} f\left(\mu+\sum_{j \in J} \delta_{y_{j}}\right)
$$


where $|J|$ denotes the number of elements of $J$. This shows that the operator $D_{y_{1}, \ldots, y_{n}}^{n}$ is symmetric in $y_{1}, \ldots, y_{n}$, and that $\left(\mu, y_{1}, \ldots, y_{n}\right) \mapsto D_{y_{1}, \ldots, y_{n}}^{n} f(\mu)$ is measurable whenever $f: \mathbf{N} \rightarrow \mathbb{R}$ is measurable.

Let $\mathbf{F}^{+}$denote the space of all bounded and measurable functions $v: \mathbb{Y} \rightarrow \mathbb{R}_{+}$. By Lemma 12.2 in [21] the Laplace functional of $\eta$ is given by

$$
\mathbb{E} \exp [-\eta(v)]=\exp \left[-\lambda\left(1-e^{-v}\right)\right], \quad v \in \mathbf{F}^{+},
$$

where $\mu(v):=\int v d \mu$ for any measure $\mu$ on $\mathbb{Y}$.

Let $\mathcal{Y}_{0}$ be the system of all measurable $B \in \mathcal{Y}$ having $\lambda(B)<\infty$. Let $\mathbf{F}_{B}^{+}$denote the space of all those $v \in \mathbf{F}^{+}$vanishing outside $B \in \mathcal{Y}$. Let $\mathbf{F}_{0}^{+}$be the space of all functions $v$ that belong to $\mathbf{F}_{B}^{+}$for some $B \in \mathcal{Y}_{0}$. Let $\mathbf{G}$ denote the space of all (bounded and measurable) functions $g: \mathbf{N} \rightarrow \mathbb{R}$ of the form

$$
g(\mu)=a_{1} e^{-\mu\left(v_{1}\right)}+\ldots+a_{n} e^{-\mu\left(v_{n}\right)},
$$

where $n \in \mathbb{N}, a_{1}, \ldots, a_{n} \in \mathbb{R}$ and $v_{1}, \ldots, v_{n} \in \mathbf{F}_{0}^{+}$.

Lemma 2.1. Relation (2.3) holds for $f, g \in \mathbf{G}$.

ProOF: By linearity it suffices to consider functions $f$ and $g$ of the form

$$
f(\mu)=\exp [-\mu(v)], \quad g(\mu)=\exp [-\mu(w)]
$$

for $v, w \in \mathbf{F}_{0}^{+}$. Then we have for $n \geq 1$ that

$$
D^{n} f(\mu)=\exp [-\mu(v)]\left(e^{-v}-1\right)^{\otimes n}
$$

where $\left(e^{-v}-1\right)^{\otimes n}\left(y_{1}, \ldots, y_{n}\right):=\prod_{i=1}^{n}\left(e^{-v\left(y_{i}\right)}-1\right)$. From (2.5) we obtain that

$$
T_{n} f=\exp \left[-\lambda\left(1-e^{-v}\right)\right]\left(e^{-v}-1\right)^{\otimes n} .
$$

Since $v \in \mathbf{F}_{0}^{+}$it follows that $T_{n} f \in \mathbf{H}_{n}, n \geq 0$. Using (2.5), we obtain that

$$
\mathbb{E} f(\eta) g(\eta)=\exp \left[-\lambda\left(1-e^{-(v+w)}\right)\right] .
$$

On the other hand we have from $(2.7)$ (putting $\lambda^{0}(1):=1$ ) that

$$
\begin{aligned}
\sum_{n=0}^{\infty} \frac{1}{n !}\langle & \left.T_{n} f, T_{n} g\right\rangle_{n} \\
& =\exp \left[-\lambda\left(1-e^{-v}\right)\right] \exp \left[-\lambda\left(1-e^{-w}\right)\right] \sum_{n=0}^{\infty} \frac{1}{n !} \lambda^{n}\left(\left(\left(e^{-v}-1\right)\left(e^{-w}-1\right)\right)^{\otimes n}\right) \\
& =\exp \left[-\lambda\left(2-e^{-v}-e^{-w}\right)\right] \exp \left[\lambda\left(\left(e^{-v}-1\right)\left(e^{-w}-1\right)\right)\right] .
\end{aligned}
$$

This equals the right-hand side of (2.8).

To extend (2.3) to general $f, g \in L^{2}\left(\mathbb{P}_{\eta}\right)$ we need two lemmas.

Lemma 2.2. The set $\mathbf{G}$ is dense in $L^{2}\left(\mathbb{P}_{\eta}\right)$. 
Proof: Let $\mathbf{W}$ be the space of all bounded measurable $g: \mathbf{N} \rightarrow \mathbb{R}$ that can be approximated in $L^{2}\left(\mathbb{P}_{\eta}\right)$ by functions in $\mathbf{G}$. This space is closed under monotone and uniformly bounded convergence and contains the constant functions. The space $\mathbf{G}$ is stable under multiplication and we denote by $\mathcal{N}^{\prime}$ the smallest $\sigma$-field on $\mathbf{N}$ such that $\mu \mapsto h(\mu)$ is measurable for all $h \in \mathbf{G}$. A well-known functional version of the monotone class theorem (see e.g. Theorem I.21 in [7]) implies that $\mathbf{W}$ contains any bounded $\mathcal{N}^{\prime}$ measurable $g$. On the other hand we have that

$$
\mu(C)=\lim _{t \rightarrow 0+} t^{-1}\left(1-e^{-t \mu(C)}\right), \quad \mu \in \mathbf{N},
$$

for any $C \in \mathcal{Y}$. Hence $\mu \mapsto \mu(C)$ is $\mathcal{N}^{\prime}$-measurable whenever $C \in \mathcal{Y}_{0}$. Since $\lambda$ is $\sigma$-finite, for any $C \in \mathcal{Y}$ there is a monotone sequence $C_{k} \in \mathcal{Y}_{0}, k \in \mathbb{N}$, with union $C$, so that $\mu \mapsto \mu(C)$ is $\mathcal{N}^{\prime}$-measurable. Hence $\mathcal{N}^{\prime}=\mathcal{N}$ and it follows that $\mathbf{W}$ contains all bounded measurable functions. But then $\mathbf{W}$ is clearly dense in $L^{2}\left(\mathbb{P}_{\eta}\right)$ and the proof of the lemma is complete.

In proving the next lemma, and repeatedly later, we need to consider factorial moment measures. For $\mu \in \mathbf{N}(\mathbb{Y})$ and for $m \in \mathbb{N}$, define the measure $\mu^{(m)}$ on $\mathbb{Y}^{m}$ by

$$
\begin{array}{r}
\mu^{(m)}(B):=\int \cdots \int \mathbf{1}_{B}\left(y_{1}, \ldots, y_{m}\right)\left(\mu-\sum_{j=1}^{m-1} \delta_{y_{j}}\right)\left(d y_{m}\right)\left(\mu-\sum_{j=1}^{m-2} \delta_{y_{j}}\right)\left(d y_{m-1}\right) \\
\ldots\left(\mu-\delta_{y_{1}}\right)\left(d y_{2}\right) \mu\left(d y_{1}\right) .
\end{array}
$$

If we can write $\mu=\sum_{i>1} \delta_{x_{i}}$, then $\mu^{(m)}(B)$ counts the number of $n$-tuples of distinct indices $\left(i_{1}, \ldots, i_{m}\right)$ such that $\left(x_{i_{1}}, \ldots, x_{i_{k}}\right)$ is in $B$, and $\mathbb{E} \eta^{(m)}(\cdot)$ is known as the $m$ th factorial moment measure of the Poisson process $\eta$. A standard tool in the analysis of Poisson driven stochastic systems is the formula

$$
\begin{aligned}
\mathbb{E} \int h\left(\eta, y_{1}, \ldots, y_{m}\right) & \eta^{(m)}\left(d\left(y_{1}, \ldots, y_{m}\right)\right) \\
& =\mathbb{E} \int h\left(\eta+\delta_{y_{1}}+\ldots+\delta_{y_{m}}, y_{1}, \ldots, y_{m}\right) \lambda^{m}\left(d\left(y_{1}, \ldots, y_{m}\right)\right),
\end{aligned}
$$

for all $h: \mathbf{N} \times \mathbb{Y}^{m} \rightarrow[-\infty, \infty]$ for which one (and then also the other) side makes sense. When $m=1$, (2.10) simplifies to the following classical formula by Mecke [25]:

$$
\mathbb{E} \int h(\eta, y) \eta(d y)=\mathbb{E} \int h\left(\eta+\delta_{y}, y\right) \lambda(d y) .
$$

In the special case where $\lambda$ is a finite, absolutely continuous measure on $\mathbb{R}^{d}$, a proof of (2.10) is given in e.g. Theorem 1.6 of [33], and the argument there can be extended to the general case. Here, and again later on, we use the fact that for arbitrary $\sigma$-finite $\lambda$, the standard proof of existence of a Poisson process $\eta$ with $\sigma$-finite intensity measure $\lambda$ (see e.g. Theorem 12.7 of [21]) shows that there is a version of this Poisson process process taking the form $\sum_{i=1}^{\eta(\mathbb{Y})} \delta_{X_{i}}$ for a sequence of $\mathbb{Y}$-valued random variables $X_{i}$.

Lemma 2.3. Suppose that $f, f^{1}, f^{2}, \ldots \in L^{2}\left(\mathbb{P}_{\eta}\right)$ satisfy $f^{k} \rightarrow f$ in $L^{2}\left(\mathbb{P}_{\eta}\right)$ as $k \rightarrow \infty$, and that $h: \mathbf{N} \rightarrow[0,1]$ is measurable. Let $n \in \mathbb{N}$, let $C \in \mathcal{Y}_{0}$ and set $B:=C^{n}$. Then

$$
\lim _{k \rightarrow \infty} \int_{B} \mathbb{E}\left[\left|D_{y_{1}, \ldots, y_{n}}^{n} f(\eta)-D_{y_{1}, \ldots, y_{n}}^{n} f^{k}(\eta)\right| h(\eta)\right] \lambda^{n}\left(d\left(y_{1}, \ldots, y_{n}\right)\right)=0
$$


Proof: By (2.4), the relation (2.12) is implied by the convergence

$$
\lim _{n \rightarrow \infty} \int_{B} \mathbb{E}\left[\left|f\left(\eta+\sum_{i=1}^{m} \delta_{y_{i}}\right)-f^{k}\left(\eta+\sum_{i=1}^{m} \delta_{y_{i}}\right)\right| h(\eta)\right] \lambda^{n}\left(d\left(y_{1}, \ldots, y_{n}\right)\right)=0
$$

for all $m \in\{0, \ldots, n\}$. For $m=0$ this is obvious. Assume $m \in\{1, \ldots, n\}$. Then the integral in (2.13) equals

$$
\begin{array}{rl}
\lambda(C)^{n-m} & \mathbb{E} \int_{C^{m}}\left|f\left(\eta+\sum_{i=1}^{m} \delta_{y_{i}}\right)-f^{k}\left(\eta+\sum_{i=1}^{m} \delta_{y_{i}}\right)\right| h(\eta) \lambda^{m}\left(d\left(y_{1}, \ldots, y_{m}\right)\right) \\
& =\lambda(C)^{n-m} \mathbb{E} \int_{C^{m}}\left|f(\eta)-f^{k}(\eta)\right| h\left(\eta-\sum_{i=1}^{n} \delta_{y_{i}}\right) \eta^{(m)}\left(d\left(y_{1}, \ldots, y_{m}\right)\right), \\
& \leq \lambda(C)^{n-m} \mathbb{E}\left|f(\eta)-f^{k}(\eta)\right| \eta^{(m)}\left(C^{m}\right),
\end{array}
$$

where we have used (2.10) to get the equality. By the Cauchy-Schwarz inequality the last expression is bounded above by

$$
\lambda(C)^{n-m}\left(\mathbb{E}\left(f(\eta)-f^{k}(\eta)\right)^{2}\right)^{1 / 2}\left(\mathbb{E}\left[\left(\eta^{(m)}\left(C^{m}\right)\right)^{2}\right]\right)^{1 / 2} .
$$

Since any Poisson variable has moments of all orders, we obtain (2.13) and hence the lemma.

Later we will also need the following direct consequence of (2.11).

Lemma 2.4. Let $f, g: \mathbf{N} \rightarrow \mathbb{R}$ be measurable functions that coinicde $\mathbb{P}_{\eta^{-}}$a.e. Then $D_{y} f(\mu)=D_{y} g(\mu)$ for $\mathbb{P}_{\eta} \otimes \lambda$-a.e. $(\mu, y)$.

Proof of Theorem 1.1: By linearity and the polarization identity

$$
4\langle f, g\rangle_{\mathbf{H}}=\langle f+g, f+g\rangle_{\mathbf{H}}-\langle f-g, f-g\rangle_{\mathbf{H}}
$$

it suffices to prove $(2.3)$ for $f=g \in L^{2}(\mathbb{P})$. By Lemma 2.2 there are $f^{k} \in \mathbf{G}, k \in \mathbb{N}$, satisfying $f^{k} \rightarrow f$ in $L^{2}\left(\mathbb{P}_{\eta}\right)$ as $k \rightarrow \infty$. By Lemma $2.1, T f^{k}, k \in \mathbb{N}$, is a Cauchy sequence in $\mathbf{H}$. Let $\tilde{f}=\left(\tilde{f}_{n}\right) \in \mathbf{H}$ be the limit, that is

$$
\lim _{k \rightarrow \infty} \sum_{n=0}^{\infty} \frac{1}{n !}\left\|T_{n} f^{k}-\tilde{f}_{n}\right\|_{n}^{2}=0 .
$$

Taking the limit in the identity $\mathbb{E} f^{k}(\eta)^{2}=\left\langle T f^{k}, T f^{k}\right\rangle_{\mathbf{H}}$ yields $\mathbb{E} f(\eta)^{2}=\langle\tilde{f}, \tilde{f}\rangle_{\mathbf{H}}$. Equation (2.14) implies that $\tilde{f}_{0}=\mathbb{E} f(\eta)=T_{0} f$. It remains to show that for any $n \geq 1$,

$$
\tilde{f}_{n}=T_{n} f \quad \lambda^{n} \text {-a.e. }
$$

Let $C \in \mathcal{Y}_{0}$ and $B:=C^{n}$. Let $\left.\lambda^{n}\right|_{B}$ be the restriction of the measure $\lambda^{n}$ to $B$. By $(2.14)$ $T_{n} f^{k}$ converges in $L^{2}\left(\left.\lambda^{n}\right|_{B}\right)$ (and hence in $L^{1}\left(\left.\lambda^{n}\right|_{B}\right)$ ) to $\tilde{f}_{n}$, while by the definition (1.3) of $T_{n}$, and the case $h \equiv 1$ of $(2.13), T_{n} f^{k}$ converges in $L^{1}\left(\left.\lambda^{n}\right|_{B}\right)$ to $T_{n} f$. Hence these $L^{1}$ limits must be the same almost everywhere, so that $\tilde{f}_{n}=T_{n} f \lambda^{n}$-a.e. on $B$. Since $\lambda$ is assumed $\sigma$-finite, this implies (2.15) and hence the theorem. 


\section{Chaos expansion}

Given functions $g_{i}: \mathbb{Y} \rightarrow \mathbb{R}$ for $i=1,2, \ldots, n$, define the tensor product function $\otimes_{i=1}^{n} g_{i}$ to be the function from $\mathbb{Y}^{n}$ to $\mathbb{R}$ which maps each $\left(y_{1}, \ldots, y_{n}\right)$ to $\prod_{i=1}^{n} g_{i}\left(y_{i}\right)$. When the functions $g_{1}, \ldots, g_{n}$ are all the same function $g$, we write $g^{\otimes n}$ for this tensor product function. This is consistent with notation used in the proof of Lemma 2.1.

For $n \in \mathbb{N}_{0}$ and $g \in L^{2}\left(\lambda^{n}\right)$ we define the multiple Wiener-Itô integral $I_{n}(g)$ of $g$ with respect to the compensated Poisson process $\hat{\eta}:=\eta-\lambda$ as follows. Set $I_{0}(c):=c$ for $c \in \mathbb{R}$. When $n \geq 1$, consider first the case where $g$ is of product form, by which we mean $g=\otimes_{i=1}^{n} g_{i}$, with each of the $g_{i}$ bounded and vanishing outside $B$ for some $B \in \mathcal{Y}_{0}$. For such $g$, set

$$
I_{n}(g):=\sum_{J \subset[n]}(-1)^{n-|J|} \eta^{(|J|)}\left(\otimes_{j \in J} g_{j}\right) \lambda^{n-|J|}\left(\otimes_{j^{\prime} \in[n] \backslash J} g_{j^{\prime}}\right),
$$

where $[n]:=\{1, \ldots, n\}$, and $|J|$ denotes the number of elements of $J$, and $\eta^{(m)}(\cdot)$ denotes integration with respect to the measure $\eta^{(m)}$ defined by $(2.9)$, while $\eta^{(0)}\left(\otimes_{j \in \emptyset} g_{j}\right):=1$ and $\lambda^{0}\left(\otimes_{j \in \emptyset} g_{j}\right):=1$. In the special case where the functions $g_{i}$ are all the same function $h$, the formula (3.1) simplifies to

$$
I_{n}\left(h^{\otimes n}\right)=\sum_{k=0}^{n}\left(\begin{array}{l}
n \\
k
\end{array}\right)(-1)^{n-k} \eta^{(k)}\left(h^{\otimes k}\right)(\lambda(h))^{n-k} .
$$

Recall $[31,17,2]$ that the Charlier polynomials $C_{n}(\lambda ; \cdot), n \in \mathbf{N}_{0}$, are a family of orthogonal polynomials for the Poisson distribution with parameter $\lambda \geq 0$ defined by

$$
C_{n}(\lambda ; x)=\sum_{k=0}^{n}\left(\begin{array}{l}
n \\
k
\end{array}\right)(-1)^{n-k} \lambda^{-k}(x)_{k}
$$

where $(x)_{j}$ is the descending factorial $x(x-1) \cdot \ldots \cdot(x-j+1)$ with $(x)_{0}$ interpreted as 1. Assume that the function $g$ in (3.1) is of the form $g=\mathbf{1}_{B_{1}}^{\otimes m_{1}} \otimes \ldots \otimes \mathbf{1}_{B_{k}}^{\otimes m_{k}}$, where $B_{1}, \ldots, B_{k} \in \mathcal{Y}_{0}$ are pairwise disjoint and $m_{1}+\ldots+m_{k}=n$. Then

$$
I_{n}(g)=\sum_{j_{1}=0}^{m_{1}} \ldots \sum_{j_{k}=0}^{m_{k}} \prod_{i=1}^{k}\left(\begin{array}{c}
m_{i} \\
j_{i}
\end{array}\right)\left(\eta\left(B_{i}\right)\right)_{j_{i}}\left(-\lambda\left(B_{i}\right)\right)^{m_{i}-j_{i}} .
$$

This can be written as

$$
I_{n}\left(\mathbf{1}_{B_{1}}^{\otimes m_{1}} \otimes \ldots \otimes \mathbf{1}_{B_{k}}^{\otimes m_{k}}\right)=\prod_{i=1}^{k} \lambda\left(B_{i}\right)^{m_{i}} C_{m_{i}}\left(\lambda\left(B_{i}\right) ; \eta\left(B_{i}\right)\right),
$$

at least if $\eta\left(B_{i}\right)<\infty$ for $i=1, \ldots, k$, an event with probability 1. Ogura [31] used this formula to define the Wiener-Itô integral for a homogeneous Poisson process on the line. Liebscher [22] generalized this approach to Poisson processes on a complete separable metric space with locally finite intensity measure.

We extend the definition of $I_{n}(g)$ by linearity to those functions $g$ which can be expressed as a finite sum of functions of product form; we shall say that such $g$ are of 
sum-product form. The extension is well defined because each term of the sum in the right hand side of (3.1) is \pm 1 times the integral of $g$ with respect to a certain measure on $\mathbb{Y}^{m}$, and hence is linear in $g$.

Let $\Sigma_{n}$ denote the set of all permutations of $[n]$, and for $g \in L^{2}\left(\lambda^{n}\right)$ define the symmetrization $\tilde{g}$ of $g$ by

$$
\tilde{g}\left(y_{1}, \ldots, y_{n}\right):=\frac{1}{n !} \sum_{\pi \in \Sigma_{n}} g\left(y_{\pi(1)}, \ldots, y_{\pi(n)}\right) .
$$

By (3.1), for $g$ of product form $I_{n}(g)$ is invariant under permutations of the functions $g_{i}$ in the tensor product. Hence $I_{n}(g)=I_{n}(\tilde{g})$ for all $g$ of sum-product form.

We shall show in Lemma 3.1 below that if $g$ and $h$ are functions of sum-product form on $\mathbb{Y}^{n}$ and on $\mathbb{Y}^{m}$ respectively, we have the isometry relation

$$
\mathbb{E} I_{m}(g) I_{n}(h)=\mathbf{1}\{m=n\} m !\langle\tilde{g}, \tilde{h}\rangle_{n}, \quad m, n \in \mathbb{N}_{0} .
$$

Since functions of sum-product form are dense in $L^{2}\left(\lambda^{n}\right)$, we can (and do) extend the definition of $I_{n}(g)$ to general $g \in L^{2}\left(\lambda^{n}\right)$ by isometry. It follows from the isometry that $I_{m}(g)=I_{m}(\tilde{g})$ for all $g \in L^{2}\left(\lambda^{m}\right)$, and that $I_{m}(g)$ is linear in $g$, and that (3.5) holds for all $g \in L^{2}\left(\lambda^{m}\right)$ and $h \in L^{2}\left(\lambda^{n}\right)$, since all these properties were already established for functions of sum-product form.

The proofs of (3.5) in the literature (see [16], [39]) assume further structure on the measure space $(\mathbb{Y}, \mathcal{Y}, \lambda)$, typically including diffuseness or continuity of $\lambda$, and in some cases, topological assumptions on $\mathbb{Y}$. We are not making such assumptions here, so we provide a new proof of (3.5), as follows. An alternative proof can be based on (3.3) and the orthogonality properties of the Charlier polynomials, see [22].

Lemma 3.1. Let $m, n \in \mathbb{N}_{0}$, and suppose $g: \mathbb{Y}^{m} \rightarrow \mathbb{R}$ and $h: \mathbb{Y}^{n} \rightarrow \mathbb{R}$ are of sum-product form. Then $I_{m}(g), I_{n}(h) \in L^{2}(\mathbb{P})$, and (3.5) holds.

ProOF: First consider $g$ and $h$ of product form. Suppose $n>0$ and $m>0$, and suppose $g=\otimes_{j=1}^{n} g_{j}$ and $h=\otimes_{k=1}^{m} h_{k}$, where all the functions $g_{j}$ and $h_{k}$ are bounded and vanish outside of some $B \in \mathcal{Y}_{0}$. Then

$$
\begin{aligned}
\mathbb{E} I_{m}(g) I_{n}(h)= & \sum_{J \subset[m]} \sum_{K \subset[n]}(-1)^{m+n-|J|-|K|}\left(\prod_{j^{\prime} \in[m] \backslash J} \lambda\left(g_{j^{\prime}}\right)\right)\left(\prod_{k^{\prime} \in[n] \backslash J} \lambda\left(g_{k^{\prime}}\right)\right) \\
& \times \mathbb{E} \eta^{(|J|)}\left(\otimes_{j \in J} g_{j}\right) \eta^{(|K|)}\left(\otimes_{k \in K} h_{k}\right) .
\end{aligned}
$$

Suppose, in each term of the preceding sum, we were to replace the expectation by $\mathbb{E}\left[\eta^{(|J|+|K|)}\left(\left(\otimes_{j \in J} g_{j}\right) \otimes\left(\otimes_{k \in K} h_{k}\right)\right)\right]$. Then the modified sum would come to zero, because by (2.10), the modified expectation comes to $\left(\prod_{j \in J} \lambda\left(\eta_{j}\right)\right)\left(\prod_{k \in K} \lambda\left(\eta_{k}\right)\right)$, so in the modified sum each term is of the form $(-1)^{m+n-|J|-|K|}\left(\prod_{j=1}^{m} \lambda\left(g_{j}\right)\right)\left(\prod_{k=1}^{n} \lambda\left(h_{j}\right)\right)$ (this also shows that (3.5) holds when one of $m$ and $n$ is 0 .) Therefore,

$$
\begin{aligned}
\mathbb{E} I_{m}(g) I_{n}(h)= & \sum_{J \subset[m]} \sum_{K \subset[n]}(-1)^{m+n-|J|-|K|}\left(\prod_{j^{\prime} \in[m] \backslash J} \lambda\left(g_{j^{\prime}}\right)\right)\left(\prod_{k^{\prime} \in[n] \backslash K} \lambda\left(g_{k^{\prime}}\right)\right) \\
& \times \mathbb{E}\left[\eta^{(|J|)}\left(\otimes_{j \in J} g_{j}\right) \eta^{(|K|)}\left(\otimes_{k \in K} h_{k}\right)-\eta^{(|J|+|K|)}\left(\left(\otimes_{j \in J} g_{j}\right) \otimes\left(\otimes_{k \in K} h_{k}\right)\right)\right] .
\end{aligned}
$$


Suppose we write the restriction of $\eta$ to $B$ as $\sum_{i=1}^{N} \delta_{X_{i}}$ (for any Poisson process this is possible, as remarked earlier). Then $\eta^{(|J|)}\left(\prod_{j \in J} g_{j}\right)$ is the sum, over all $|J|$-tuples of distinct points $X_{i}, i \in[N]$, of the product of the values of $g_{j}$ at those points. Similarly, $\eta^{(|K|)}\left(\otimes_{k \in K} h_{k}\right)$ is the sum, over all $|K|$-tuples of distinct points $X_{i}$, of the product of the values of $h_{j}$ at those points. When multiplied together, the $j$-tuple and the $k$-tuple need to have at least one element in common for this product to be different from the corresponding term in $\eta^{(|J|+|K|)}\left(\left(\otimes_{j \in J} g_{j}\right) \otimes\left(\otimes_{k \in K} h_{k}\right)\right)$. For example, if $J=K=\{1,2\}$ we have

$$
\begin{aligned}
\eta^{(2)}\left(g_{1} \otimes g_{2}\right) & \eta^{(2)}\left(h_{1} \otimes h_{2}\right)-\eta^{(4)}\left(g_{1} \otimes g_{2} \otimes h_{1} \otimes h_{2}\right) \\
= & \eta^{(3)}\left(g_{1} h_{1} \otimes g_{2} \otimes h_{2}+g_{1} h_{2} \otimes g_{2} \otimes h_{1}+g_{2} h_{1} \otimes g_{1} \otimes h_{2}+g_{2} h_{2} \otimes g_{1} \otimes h_{1}\right) \\
& +\eta^{(2)}\left(g_{1} h_{1} \otimes g_{2} h_{2}+g_{1} h_{2} \otimes g_{2} h_{1}\right)
\end{aligned}
$$

where the product $g_{i} h_{j}$ is defined pointwise, that is $g_{i} h_{j}(y):=g_{i}(y) h_{j}(y)$ for $y \in \mathbb{Y}$. In general, for each matching (bijection) $\varphi$ of a nonempty subset $\left\{j_{1}, \ldots, j_{\alpha}\right\}$ of $J$ to a subset of $K$, writing $k_{i}$ for $\varphi\left(j_{i}\right)$ we get a contribution to the expression inside the expectation in the $(J, K)$ th term of (3.6) which is of the form

$$
\eta^{(|J|+|K|-\alpha)}\left(\left(\otimes_{i=1}^{\alpha} g_{j_{i}} h_{k_{i}}\right) \otimes\left(\otimes_{j \in J \backslash\left\{j_{1}, \ldots, j_{\alpha}\right\}} g_{j}\right) \otimes\left(\otimes_{k \in K \backslash\left\{k_{1}, \ldots, k_{\alpha}\right\}} h_{k}\right)\right)
$$

so that when one takes the expectation using (2.10), and multiplies by the remaining factors of $\lambda\left(g_{j^{\prime}}\right) \lambda\left(h_{k^{\prime}}\right)$ appearing in this term of the right hand side of (3.6), one ends up with a contribution of

$$
(-1)^{m+n-|J|-|K|}\left(\prod_{i=1}^{\alpha} \lambda\left(g_{j_{i}} h_{k_{i}}\right)\right) \times\left(\prod_{j \in[m] \backslash\left\{j_{1}, \ldots, j_{\alpha}\right\}} \lambda\left(g_{j}\right)\right) \times\left(\prod_{k \in[n] \backslash\left\{k_{1}, \ldots, k_{\alpha}\right\}} \lambda\left(h_{k}\right)\right)
$$

which depends on $J$ and $K$ only through the sign factor. Writing $J=\left\{j_{1}, \ldots, j_{\alpha}\right\} \cup J^{\prime}$ and $K=\left\{k_{1}, \ldots, k_{\alpha}\right\} \cup K^{\prime}$, we have that the total contribution to the right hand side of (3.6) from a given matching $\varphi$ is given by

$$
\begin{aligned}
& \left(\prod_{i=1}^{\alpha} \lambda\left(g_{j_{i}} h_{k_{i}}\right)\right) \times\left(\prod_{j \in[m] \backslash\left\{j_{1}, \ldots, j_{\alpha}\right\}} \lambda\left(g_{j}\right)\right) \times\left(\prod_{k \in[n] \backslash\left\{k_{1}, \ldots, k_{\alpha}\right\}} \lambda\left(h_{k}\right)\right) \\
& \quad \times \sum_{J^{\prime} \subset[m] \backslash\left\{j_{1}, \ldots, j_{\alpha}\right\}} \sum_{K^{\prime} \subset[n] \backslash\left\{k_{1}, \ldots, k_{\alpha}\right\}}(-1)^{m+n-\left|J^{\prime}\right|-\left|K^{\prime}\right|-2 \alpha}
\end{aligned}
$$

and this comes to zero, except in the case where $\alpha=m=n$.

Hence, all matchings contribute zero to (3.6) unless $m=n$, and for this case there are $n$ ! matchings $\varphi$ having $\alpha=n$, namely the permutations of $[n]$, so that

$$
\mathbb{E}\left[I_{n}(g) I_{n}(h)\right]=\sum_{\varphi \in \Sigma_{n}} \prod_{i=1}^{n} \lambda\left(g_{i} h_{\varphi(i)}\right)=\sum_{\varphi \in \Sigma_{n}} \prod_{i=1}^{n}\left\langle g_{i}, h_{\varphi(i)}\right\rangle_{n}
$$

With the symmetrization $\tilde{g}$ defined at (3.4), we have by linearity that

$$
\mathbb{E} I_{n}(\tilde{g}) I_{n}(\tilde{h})=n !^{-2} \sum_{\pi \in \Sigma_{n}} \sum_{\sigma \in \Sigma_{n}} \mathbb{E}\left[I_{n}\left(\otimes_{i=1}^{n} g_{\pi(i)}\right) I_{n}\left(\otimes_{j=1}^{n} h_{\sigma(j)}\right)\right]=\sum_{\varphi \in \Sigma_{n}} \prod_{i=1}^{n}\left\langle g_{i}, h_{\varphi(i)}\right\rangle_{n}
$$


whereas

$$
\langle\tilde{g}, \tilde{h}\rangle_{n}=n !^{-2} \sum_{\pi \in \Sigma_{n}} \sum_{\sigma \in \Sigma_{n}}\left\langle g_{\pi(i)}, h_{\sigma(j)}\right\rangle_{n}=n !^{-1} \sum_{\varphi \in \Sigma_{n}}\left\langle g_{i}, h_{\varphi(i)}\right\rangle_{n}
$$

so that (3.5) holds for this case. We can then extend by linearity to all $f$ and $g$ of sum-product form.

The proof of Theorem 1.3 requires the following key lemma.

Lemma 3.2. Let $f(\mu):=e^{-\mu(v)}, \mu \in \mathbf{N}(\mathbb{Y})$, where $v: \mathbb{Y} \rightarrow \mathbb{R}_{+}$is a measurable function vanishing outside a set $B \in \mathcal{Y}_{0}$. Then $(1.9)$ holds $\mathbb{P}$-a.s. and in $L^{2}(\mathbb{P})$.

Proof: By (2.5) and (2.7) the right-hand side of (1.9) equals the formal sum

$$
I:=\exp \left[-\lambda\left(1-e^{-v}\right)\right]+\exp \left[-\lambda\left(1-e^{-v}\right)\right] \sum_{n=1}^{\infty} \frac{1}{n !} I_{n}\left(\left(e^{-v}-1\right)^{\otimes n}\right) .
$$

The function $\left(e^{-v}-1\right)^{\otimes n}$ is of product form. Using the pathwise definition (3.2) we obtain that almost surely

$$
\begin{aligned}
I & =\exp \left[-\lambda\left(1-e^{-v}\right)\right] \sum_{n=0}^{\infty} \frac{1}{n !} \sum_{k=0}^{n}\left(\begin{array}{l}
n \\
k
\end{array}\right) \eta^{(k)}\left(\left(e^{-v}-1\right)^{\otimes k}\right)\left(\lambda\left(1-e^{-v}\right)\right)^{n-k} \\
& =\exp \left[-\lambda\left(1-e^{-v}\right)\right] \sum_{k=0}^{\infty} \frac{1}{k !} \eta^{(k)}\left(\left(e^{-v}-1\right)^{\otimes k}\right) \sum_{n=k}^{\infty} \frac{1}{(n-k) !}\left(\lambda\left(1-e^{-v}\right)\right)^{n-k} \\
& =\sum_{k=0}^{N} \frac{1}{k !} \eta^{(k)}\left(\left(e^{-v}-1\right)^{\otimes k}\right)
\end{aligned}
$$

where $N:=\eta(B)$. Writing $\delta_{X_{1}}+\ldots+\delta_{X_{N}}$ for the restriction of $\eta$ to $B$, we have almost surely that

$$
I=\sum_{J \subset\{1, \ldots, N\}} \prod_{i \in J}\left(e^{-v\left(X_{i}\right)}-1\right)=\prod_{i=1}^{N} e^{-v\left(X_{i}\right)}=e^{-\eta(v)},
$$

and hence (1.9) holds with almost sure convergence of the series. To demonstrate that convergence also holds in $L^{2}(\mathbb{P})$, let the partial sum $I(m)$ be given by the right hand side (3.7) with the series terminated at $n=m$. Then since $\lambda\left(1-e^{-v}\right)$ is nonnegative and $\left|1-e^{-v(y)}\right| \leq 1$ for all $y$, a similar argument to (3.8) yields

$$
\begin{aligned}
|I(m)| & \leq \sum_{k=0}^{\min (N, m)} \frac{1}{k !}\left|\eta^{(k)}\left(\left(e^{-v}-1\right)^{\otimes k}\right)\right| \\
& \leq \sum_{k=0}^{N} \frac{N(N-1) \cdots(N-k+1)}{k !}=2^{N} .
\end{aligned}
$$

Since $2^{N}$ has finite moments of all orders, by dominated convergence the series (3.7) (and hence (1.9)) converges in $L^{2}(\mathbb{P})$. 
Proof of Theorem 1.3: Let $f \in L^{2}\left(\mathbb{P}_{\eta}\right)$ and define $T_{n} f$ for $n \in \mathbb{N}_{0}$ by (1.3). By (3.5) and Theorem 1.1,

$$
\sum_{n=0}^{\infty} \mathbb{E}\left(\frac{1}{n !} I_{n}\left(T_{n} f\right)\right)^{2}=\sum_{n=0}^{\infty} \frac{1}{n !}\left\|T_{n} f\right\|_{n}^{2}=\mathbb{E} f(\eta)^{2}<\infty .
$$

Hence the infinite series of orthogonal terms

$$
X:=\sum_{n=0}^{\infty} \frac{1}{n !} I_{n}\left(T_{n} f\right)
$$

converges in $L^{2}(\mathbb{P})$. Let $h \in \mathbf{G}$, where $\mathbf{G}$ was defined at (2.6). By Lemma 3.2 and linearity of $I_{n}(\cdot)$ the sum $\sum_{n=0}^{\infty} \frac{1}{n !} I_{n}\left(T_{n} h\right)$ converges in $L^{2}(\mathbb{P})$ to $h(\eta)$. Using $(3.5)$ followed by Theorem 1.1 yields

$$
\mathbb{E}(h(\eta)-X)^{2}=\sum_{n=0}^{\infty} \frac{1}{n !}\left\|T_{n} h-T_{n} f\right\|_{n}=\mathbb{E}(f(\eta)-h(\eta))^{2} .
$$

Hence if $\mathbb{E}(f(\eta)-h(\eta))^{2}$ is small, then so is $\mathbb{E}(f(\eta)-X)^{2}$. Since $\mathbf{G}$ dense in $L^{2}\left(\mathbb{P}_{\eta}\right)$ by Lemma 2.2, it follows that $f(\eta)=X$ almost surely.

To prove the uniqueness, suppose that also $g_{n} \in \mathbf{H}_{n}$ for $n \in \mathbb{N}_{0}$ are such that $\sum_{n=0}^{\infty} \frac{1}{n !} I_{n}\left(g_{n}\right)$ converges in $L^{2}(\mathbb{P})$ to $f(\eta)$. By taking expectations we must have $g_{0}=$ $\mathbb{E} f(\eta)=T_{0} f$. For $n \geq 1$ and $h \in \mathbf{H}_{n}$, by (3.5) and (1.9) we have

$$
\mathbb{E} f(\eta) I_{n}(h)=\mathbb{E} I_{n}\left(T_{n} f\right) I_{n}(h)=n !\left\langle T_{n} f, h\right\rangle_{n}
$$

and similarly with $T_{n} f$ replaced by $g_{n}$, so that $\left\langle T_{n} f-g_{n}, h\right\rangle_{n}=0$. Putting $h=T_{n} f-g_{n}$ gives $\left\|T_{n} f-g_{n}\right\|_{n}=0$ for each $n$, completing the proof of the theorem.

We proceed with proving that the pathwise defined difference operator $D_{y}$ coincides with a derivative operator acting on square integrable $\sigma(\eta)$-measurable random variables. For $n \in \mathbb{N}, f \in \mathbf{H}_{n}$, and $y \in \mathbb{Y}$ we define $f_{y}: \mathbb{Y}^{n-1} \rightarrow \mathbb{R}$ by $f_{y}\left(y_{1}, \ldots, y_{n-1}\right):=$ $f\left(y_{1}, \ldots, y_{n-1}, y\right)$. Whenever $f_{y} \in L^{2}\left(\lambda^{n-1}\right)$ (which is the case for $\lambda$-a.e. $y$ ) we define $I_{n-1} f(y):=I_{n-1}\left(f_{y}\right)$. Otherwise we set $I_{n-1} f(y):=0$. We choose a version of $I_{n-1} f(y)$ that is jointly measurable in $\omega$ and $y$. Strictly speaking, we claim that there is a $h \in L^{2}\left(\mathbb{P} \otimes \lambda^{n}\right)$ and a $B \in \mathcal{Y}$ such that $\lambda(\mathbb{Y} \backslash B)=0$ and $I_{n-1} f(y)=h(\cdot, y) \mathbb{P}$-a.s. for any $y \in B$. If $f$ was a function of sum-product form then by using (3.1) one could see directly that $I_{n-1} f$ was jointly measurable. In general we approximate $f$ in $L^{2}\left(\lambda^{n}\right)$ by symmetric functions $f^{k}, k \in \mathbb{N}$, of sum-product form. Since by (3.5)

$$
\int \mathbb{E}\left|I_{n-1} f^{k}(y)-I_{n-1} f^{l}(y)\right|^{2} \lambda(d y)=\int\left\|f_{y}^{k}-f_{y}^{l}\right\|_{n-1}^{2} \lambda(d y)=\left\|f^{k}-f^{l}\right\|_{n}^{2}, \quad k, l \in \mathbb{N},
$$

we can take $h$ as the $L^{2}$-limit of the Cauchy sequence $I_{n-1} f^{k}(y)$. Next we can choose $B_{1} \in \mathcal{Y}$ and a subsequence $J \subset \mathbb{N}$ such that $\lambda\left(\mathbb{Y} \backslash B_{1}\right)=0$ and $\mathbb{E}\left|I_{n-1} f^{k}(y)-h(\cdot, y)\right|^{2} \rightarrow 0$ as $k \rightarrow \infty$ along $J$ for all $y \in B_{1}$. On the other hand we may also choose $B_{2} \in \mathcal{Y}$ and a subsequence $J^{\prime} \subset J$ such that $\lambda\left(\mathbb{Y} \backslash B_{2}\right)=0$ and $\left\|f_{y}^{k}-f_{y}\right\|_{n-1}^{2} \rightarrow 0$ as $k \rightarrow \infty$ along $J^{\prime}$ 
for all $y \in B_{2}$. But then $\mathbb{E}\left|I_{n-1} f^{k}(y)-I_{n-1} f(y)\right|^{2} \rightarrow 0$ as $k \rightarrow \infty$ along $J^{\prime}$, implying that $I_{n-1} f(y)=h(\cdot, y) \mathbb{P}$-a.s. for any $y \in B_{1} \cap B_{2}$.

Given $f \in L^{2}\left(\mathbb{P}_{\eta}\right)$, define $f_{n}=\frac{1}{n !} T_{n} f \in \mathbf{H}_{n}$, so by Theorem 1.3,

$$
f(\eta)=\sum_{n=0}^{\infty} I_{n}\left(f_{n}\right)
$$

is the chaotic expansion of $f$ (with $L^{2}(\mathbb{P})$ convergence). We then define

$$
D_{y}^{\prime} f(\eta):=\sum_{n=1}^{\infty} n I_{n-1} f_{n}(y)
$$

provided that

$$
\sum_{n=1}^{\infty} n \cdot n ! \int f_{n}^{2} d \lambda^{n}<\infty
$$

In this case, by (3.5),

$$
\mathbb{E} \int\left(D_{y}^{\prime} f(\eta)\right)^{2} \lambda(d y)=\int\left(\sum_{n=1}^{\infty} n \cdot n !\left\|\left(f_{n}\right)_{y}\right\|_{n-1}^{2}\right) \lambda(d y)=\sum_{n=1}^{\infty} n \cdot n ! \int\left\|f_{n}\right\|_{n}^{2} .
$$

Therefore we can interpret $D^{\prime}$ as a linear derivative operator from $L^{2}\left(\mathbb{P}_{\eta}\right)$ to $L^{2}\left(\mathbb{P}_{\eta} \otimes \lambda\right)$, see e.g. [30]. The following result generalizes Theorem 6.5 in [17] (see also Theorem 6.2 in $[30])$.

Theorem 3.3. Let $f \in L^{2}\left(\mathbb{P}_{\eta}\right)$ have a chaotic expansion (3.9) satisfying (3.11). Then

$$
D_{y} f(\eta)=D_{y}^{\prime} f(\eta) \quad \mathbb{P} \text {-a.s., } \lambda \text {-a.e. } y \text {. }
$$

Before proving Theorem 3.3, it is convenient to introduce the dual operator of $D^{\prime}$. Let $h \in L^{2}\left(\mathbb{P}_{\eta} \otimes \lambda\right)$. Then $h(\cdot, y) \in L^{2}\left(\mathbb{P}_{\eta}\right)$ for $\lambda$-a.e. $y$. Define

$$
\tilde{h}_{n}\left(y_{1}, \ldots, y_{n+1}\right)=\frac{1}{(n+1) !} \sum_{i=1}^{n+1} \mathbb{E} D_{y_{1}, \ldots, y_{i-1}, y_{i+1}, \ldots, y_{n}}^{n} f\left(\eta, y_{i}\right) .
$$

From Theorem 1.1 we obtain that $\tilde{h}_{n} \in \mathbf{H}_{n+1}$ and we can define the Kabanov-Skorohod integral $[11,18,40,19]$ of $h$, denoted $\delta(h)$, by

$$
\delta(h):=\sum_{n=0}^{\infty} I_{n+1}\left(\tilde{h}_{n}\right)
$$

which converges in $L^{2}(\mathbb{P})$ provided that

$$
\sum_{n=0}^{\infty}(n+1) ! \int \tilde{h}_{n}^{2} d \lambda^{n+1}<\infty .
$$

The following duality relation is a special case of Proposition 4.2 in [30] applying to general Fock spaces. We give the short proof for completeness. 
Proposition 3.4. Let $f \in L^{2}\left(\mathbb{P}_{\eta}\right)$ have a chaotic expansion (3.9) satisfying (3.11) and let $h \in L^{2}\left(\mathbb{P}_{\eta} \otimes \lambda\right)$ be such that (3.15) holds. Then

$$
\mathbb{E} \int D_{y}^{\prime} f(\eta) h(\eta, y) \lambda(d y)=\mathbb{E} f(\eta) \delta(h) .
$$

Proof: For $y \in \mathbb{Y}$ with $h(\cdot, y) \in L^{2}\left(\mathbb{P}_{\eta}\right)$, the function $T_{n} h(\cdot, y) \in \mathbf{H}_{n}$ is defined, and by Theorem 1.3 the sum $\sum_{n=0}^{\infty} \frac{1}{n !} I_{n}\left(T_{n} h(\cdot, y)\right)$, converges in $L^{2}(\mathbb{P})$ to $h(\eta, y)$. Also, by the explicit formula (2.4), the function $\left(y_{1}, \ldots, y_{n}, y\right) \mapsto \frac{1}{n !} T_{n} h(\cdot, y)\left(y_{1}, \ldots, y_{n}\right)$ is measurable. Moreover $\tilde{h}_{n}$, given by (3.13), is the symmetrization of this function, as defined at (3.4). By (3.5),

$$
\mathbb{E} \int D_{y}^{\prime} f(\eta) h(\eta, y) \lambda(d y)=\sum_{n=1}^{\infty} \int\left\langle\left(f_{n}\right)_{y}, T_{n-1} h(\cdot, y)\right\rangle_{n-1} \lambda(d y)=\sum_{n=1}^{\infty} n !\left\langle f_{n}, \tilde{h}_{n-1}\right\rangle_{n},
$$

where we have used the fact that $f_{n}$ is a symmetric function. By definition (3.14) and (3.5), the last series coincides with $\mathbb{E} f(\eta) \delta(h)$.

Proof of Theorem 3.3: First consider the case with $f(\mu)=e^{-\mu(v)}$ with $v \in \mathbf{F}_{0}^{+}$. Then $n ! f_{n}=T_{n} f$ is given by (2.7). Given $n \in \mathbb{N}$,

$$
n \cdot n ! \int f_{n}^{2} d \lambda^{n}=\frac{1}{(n-1) !} \exp \left[2 \lambda\left(e^{-v}-1\right)\right]\left(\lambda\left(\left(e^{-v}-1\right)^{2}\right)\right)^{n}
$$

which is summable in $n$, so (3.11) holds in this case. Also, in this case, $D_{y} f(\eta)=$ $\left(e^{v(y)}-1\right) f(\eta)$ by $(1.1)$, while $\left(f_{n}\right)_{y}=\left(e^{-v(y)}-1\right) n^{-1} f_{n-1}$ so that by $(3.10)$,

$$
D_{y}^{\prime} f(\eta)=\sum_{n=1}^{\infty}\left(e^{-v(y)}-1\right) I_{n-1}\left(f_{n-1}\right)=\left(e^{-v(y)}-1\right) f(\eta)
$$

where the last inequality is from Lemma 3.2 again. Thus (3.12) holds for $f$ of this form. By linearity this extends to all elements of $\mathbf{G}$.

Let us now consider the general case. Choose $g_{k} \in \mathbf{G}, k \in \mathbb{N}$, such that $g_{k} \rightarrow f$ in $L^{2}\left(\mathbb{P}_{\eta}\right)$ as $k \rightarrow \infty$, see Lemma 2.2. Define $h \in L^{2}\left(\mathbb{P}_{\eta} \otimes \lambda\right)$ by $h(\mu, y):=h^{\prime}(\mu) \mathbf{1}_{B}(y)$, where $h^{\prime}$ is as in Lemma 3.2 and $B \in \mathcal{Y}_{0}$. From Lemma 3.2 it is easy to see that (3.15) holds. Therefore we obtain from Proposition 3.4 and the linearity of the operator $D^{\prime}$ that

$$
\mathbb{E} \int\left(D_{y}^{\prime} f(\eta)-D_{y}^{\prime} g_{k}(\eta)\right) h(\eta, y) \lambda(d y)=\mathbb{E}\left(f(\eta)-g_{k}(\eta)\right) \delta(h) \rightarrow 0 \quad \text { as } k \rightarrow \infty .
$$

On the other hand,

$$
\mathbb{E} \int\left(D_{y} f(\eta)-D_{y} g_{k}(\eta)\right) h(\eta, y) \lambda(d y)=\int_{B} \mathbb{E}\left[\left(D_{y} f(\eta)-D_{y} g_{k}(\eta)\right) h^{\prime}(\eta)\right] \lambda(d y),
$$

and by the case $n=1$ of Lemma 2.3 , this tends to zero as $k \rightarrow \infty$. Since $D_{y}^{\prime} g_{k}=D_{y} g_{k}$ a.s. for $\lambda$-a.e. $y$ we obtain from (3.17) that

$$
\mathbb{E} \int\left(D_{y}^{\prime} f\right) h(\eta, y) \lambda(d y)=\mathbb{E} \int\left(D_{y} f(\eta)\right) h(\eta, y) \lambda(d y) .
$$


By Lemma 2.2, the linear combinations of the functions $h$ considered above are dense in $L^{2}\left(\mathbb{P}_{\eta} \otimes \lambda\right)$, and by linearity (3.18) carries through to $h$ in this dense class of functions too, so we may conclude that the assertion (3.12) holds.

Next we derive a pathwise interpretation of the Kabanov-Skorohod integral. For $h \in$ $L^{1}\left(\mathbb{P}_{\eta} \otimes \lambda\right)$ we define

$$
\delta^{\prime}(h):=\int h\left(\eta-\delta_{y}, y\right) \eta(d y)-\int h(\eta, y) \lambda(d y) .
$$

It turns out that the Kabanov-Skorohod integral and the operator $\delta^{\prime}$ coincide on the intersection of their domains:

Theorem 3.5. Let $h \in L^{1}\left(\mathbb{P}_{\eta} \otimes \lambda\right) \cap L^{2}\left(\mathbb{P}_{\eta} \otimes \lambda\right)$ and assume that (3.15) holds. Then $\delta(h)=\delta^{\prime}(h) \mathbb{P}$-a.s.

Proof: The Mecke equation (2.11) shows that $\mathbb{E} \int\left|h\left(\eta-\delta_{y}, y\right)\right| \eta(d y)<\infty$ as well as

$$
\mathbb{E} \int D_{y} f(\eta) h(\eta, y) \lambda(d y)=\mathbb{E} f(\eta) \delta^{\prime}(h),
$$

whenever $f: \mathbf{N} \rightarrow \mathbb{R}$ is measurable and bounded. Therefore we obtain from Theorem 3.3 and Proposition 3.4 that $\mathbb{E} f(\eta) \delta^{\prime}(h)=\mathbb{E} f(\eta) \delta(h)$ provided that $f$ satisfies (3.11). By Lemma 2.2 the space of such bounded functions is dense in $L^{2}\left(\mathbb{P}_{\eta}\right)$, so we may conclude that the assertion holds.

The duality relation (3.20) was observed in [37], at least in case $\lambda$ is diffuse. Therefore Theorem 3.5 is implicit in this work.

\section{Variance inequalities}

In this section we prove Theorem 1.2, and also give a further set of variance inequalities in Theorem 4.2. To help us identify the cases where these equalities are strict, we first give a criterion, in terms of the difference operator, for the chaotic expansion (3.9) of a function $f \in L^{2}\left(\mathbb{P}_{\eta}\right)$ to terminate after $k$ steps.

Proposition 4.1. Suppose $f \in L^{2}\left(\mathbb{P}_{\eta}\right)$ and $k \in \mathbb{N}_{0}$. Then $f$ satisfies

$$
f(\eta)=I_{0}\left(f_{0}\right)+\ldots+I_{k}\left(f_{k}\right) \quad \mathbb{P} \text {-a.s. }
$$

for some $f_{j} \in \mathbf{H}_{j}, 0 \leq j \leq k$, if and only if $D_{y_{1}, \ldots, y_{k+1}}^{k+1} f(\eta)=0$ almost surely for $\lambda^{k+1}$-a.e. $\left(y_{1}, \ldots, y_{k+1}\right)$, in which case $f_{0}=\mathbb{E} f(\eta)$ and $f_{j}$ and $T_{j} f$ are equal $\lambda^{j}$-a.e. for $j=1, \ldots, k$.

Proof: First suppose $D_{y_{1}, \ldots, y_{k+1}}^{k+1} f(\eta)=0$ almost surely for $\lambda^{k+1}$-a.e. $\left(y_{1}, \ldots, y_{k+1}\right)$. Then given such $y_{1}, \ldots, y_{k+1}$, for any $m \in \mathbb{N}$ with $m \geq k+2$ we have almost surely $D_{y_{1}, \ldots, y_{m}}^{m}(f)=0$ for any $y_{k+2}, \ldots, y_{m}$, and taking expectations shows that $T^{m} f\left(y_{1}, \ldots, y_{m}\right)$ for any $m \geq k+1$ and $\lambda^{m}$-a.e. $\left(y_{1}, \ldots, y_{m}\right)$. Applying (1.9) yields (4.1).

For the converse implication, we assume that (4.1) holds for $k \geq 1$. (The case $k=0$ follows from Lemma 2.4.) By the uniqueness part of Theorem 1.3 we then have $\left\|T_{m} f\right\|_{m}^{2}=$ 0 for all $m \geq k+1$ and hence

$$
\mathbb{E} D_{y_{1}, \ldots, y_{n}}^{n} D_{x_{1}, \ldots, x_{k}}^{k} f(\eta)=0 \quad \lambda^{n} \text {-a.e. }\left(y_{1}, \ldots, y_{n}\right), \lambda^{k} \text {-a.e. }\left(x_{1}, \ldots, x_{k}\right)
$$


for all $n \geq 1$. Applying (1.4) to the function $D_{x_{1}, \ldots, x_{k}}^{k} f$ gives

$$
\mathbb{E}\left(D_{x_{1}, \ldots, x_{k}}^{k} f(\eta)\right)^{2}=\left(\mathbb{E} D_{x_{1}, \ldots, x_{k}}^{k} f(\eta)\right)^{2} \quad \lambda^{k} \text {-a.e. }\left(x_{1}, \ldots, x_{k}\right) .
$$

Since Jensen's inequality $\mathbb{E} X^{2} \geq(\mathbb{E} X)^{2}$ is an equality iff $X$ is a.s. constant, we obtain that

$$
D_{x_{1}, \ldots, x_{k}}^{k} f(\eta)=\mathbb{E} D_{x_{1}, \ldots, x_{k}}^{k} f(\eta) \quad \mathbb{P} \text {-a.s., } \lambda^{k} \text {-a.e. }\left(x_{1}, \ldots, x_{k}\right) .
$$

By Lemma 2.4 and Fubini's theorem we get $D_{x_{1}, \ldots, x_{k+1}}^{k+1} f(\eta)=D_{x_{k+1}}^{1} D_{x_{1}, \ldots, x_{k}}^{k} f(\eta)=0$ almost surely for $\lambda^{k+1}$-a.e. $\left(x_{1}, \ldots, x_{k+1}\right)$.

For the final part, assume (4.1) is true. By the uniqueness part of Theorem 1.3 we have $\left(\lambda^{j}\right.$-a.e. $)$ that $T_{j} f=f_{j}$ for $j \leq k$, as asserted.

Next we use Theorem 1.1 to prove a series of lower and upper variance bounds associated with the truncated series (1.4). The first of these upper bounds, i.e. the case $k=1$ of (4.2) below, is the Poincaré inequality (1.8) and, as remarked earlier, is also a special case of Theorem 1.2.

Theorem 4.2. Let $f \in L^{2}\left(\mathbb{P}_{\eta}\right)$ and $k \in \mathbb{N}$. Then

$$
\sum_{n=1}^{k} \frac{1}{n !}\left\|T_{n} f\right\|_{n}^{2} \leq \operatorname{Var}[f(\eta)] \leq\left(\sum_{n=1}^{k-1} \frac{1}{n !}\left\|T_{n} f\right\|_{n}^{2}\right)+\frac{1}{k !} \mathbb{E}\left\|D^{k} f(\eta)\right\|_{k}^{2},
$$

with an empty sum interpreted as zero. Both inequalities are strict unless $D_{y_{1}, \ldots, y_{k+1}}^{k+1} f(\eta)=$ 0 almost surely for $\lambda^{k+1}$-a.e. $\left(y_{1}, \ldots, y_{k+1}\right)$, in which case both inequalities are equalities.

Proof: The first inequality follows directly from (1.4). To prove the second, write (1.4) as

$$
\begin{aligned}
\operatorname{Var}[f(\eta)] & =\left(\sum_{n=1}^{k-1} \frac{1}{n !}\left\|T_{n} f\right\|_{n}^{2}\right)+\frac{1}{k !} \int\left(\mathbb{E} D_{y_{1}, \ldots, y_{k}}^{k} f(\eta)\right)^{2} \lambda^{k}\left(d\left(y_{1}, \ldots, y_{k}\right)\right) \\
& +\sum_{n=k+1}^{\infty} \frac{1}{n !} \iint\left(\mathbb{E} D_{y_{k+1}, \ldots, y_{n}}^{n-k} D_{y_{1}, \ldots, y_{k}}^{k} f(\eta)\right)^{2} \lambda^{n-k}\left(d\left(y_{k+1}, \ldots, y_{n}\right)\right) \lambda^{k}\left(d\left(y_{1}, \ldots, y_{k}\right)\right) .
\end{aligned}
$$

By relabelling $n-k$ as $m$ for $n>k$ and $y_{\ell}$ as $x_{\ell-k}$ for $\ell>k$, it follows that

$$
\begin{aligned}
\operatorname{Var}[f(\eta)] & \leq\left(\sum_{n=1}^{k-1} \frac{1}{n !}\left\|T_{n} f\right\|_{n}^{2}\right)+\frac{1}{k !} \int\left[\left(\mathbb{E} D_{y_{1}, \ldots, y_{k}}^{k} f(\eta)\right)^{2}\right. \\
& \left.+\sum_{m=1}^{\infty} \frac{1}{m !} \int\left(\mathbb{E} D_{x_{1}, \ldots, x_{m}}^{m} D_{y_{1}, \ldots, y_{k}}^{k} f(\eta)\right)^{2} \lambda^{m}\left(d\left(x_{1}, \ldots, x_{m}\right)\right)\right] \lambda^{k}\left(d\left(y_{1}, \ldots, y_{k}\right)\right) .
\end{aligned}
$$

Assuming without loss of generality that $\mathbb{E} \int\left(D_{y_{1}, \ldots, y_{k}}^{k} f(\eta)\right)^{2} \lambda^{k}\left(d\left(y_{1}, \ldots, y_{k}\right)\right)<\infty$ (else the right hand side of (4.2) is infinite), we can apply (1.4) to the function $D_{y_{1}, y_{2}, \ldots, y_{k}}^{k} f$ for $\lambda^{k}$-a.e. $\left(y_{1}, \ldots, y_{k}\right)$, thereby simplifying the expression inside [.] above, to obtain

$$
\operatorname{Var}[f(\eta)] \leq\left(\sum_{n=1}^{k-1} \frac{1}{n !}\left\|T_{n} f\right\|_{k}^{2}\right)+\frac{1}{k !} \int \mathbb{E}\left(D_{y_{1}, \ldots, y_{k}}^{k} f(\eta)\right)^{2} \lambda^{k}\left(d\left(y_{1}, \ldots, y_{k}\right)\right)
$$


which yields (4.2).

Assume now that one of the inequalities in (4.2) is an equality. Then $\left\|T_{n} f\right\|_{n}=0$ for all $n>k$. Therefore we obtain from (1.9) and (3.5) that (4.1) holds for some $f_{j} \in \mathbf{H}_{j}$, $j=1, \ldots, k$. Hence, by Proposition $4.1, D_{y_{1}, \ldots, y_{k+1}}^{k+1} f(\eta)=0$ almost surely for $\lambda^{k+1}$-a.e. $\left(y_{1}, \ldots, y_{k+1}\right)$,

Assume, conversely, that $D_{y_{1}, \ldots, y_{k+1}}^{k+1} f(\eta)=0$ almost surely for $\lambda^{k+1}$-a.e. $\left(y_{1}, \ldots, y_{k+1}\right)$. Then by Proposition 4.1, (4.1) holds for some $f_{j} \in \mathbf{H}_{j}, j=1, \ldots, k$. Therefore, by the uniqueness part of Theorem 1.3 we have $\left(\lambda^{j}\right.$-a.e. $)$ that $T_{j} f=0$ for $j>k$, so that $\left\|T_{n} f\right\|_{n}=0$ for $n>k$ and hence we have equalities in (4.2).

Proof of Theorem 1.2: For any $n \in \mathbb{N}$ we abbreviate $a_{n}:=\left\|\mathbb{E} D^{n} f(\eta)\right\|_{n}^{2}$. Using (1.4) for $D_{y_{1}, \ldots, y_{n}}^{n} f$ we get

$$
\mathbb{E} \int\left(D_{y_{1}, \ldots, y_{n}}^{n} f(\eta)\right)^{2} \lambda^{n}\left(d\left(y_{1}, \ldots, y_{n}\right)\right)=\sum_{m=0}^{\infty} \frac{a_{m+n}}{m !}=\sum_{m=n}^{\infty} \frac{a_{m}}{(m-n) !} .
$$

Hence, using (1.4) again, we have that (1.7) is equivalent to

$$
\sum_{n=1}^{2 k} \sum_{m=n}^{\infty} \frac{(-1)^{n+1}}{n !} \frac{a_{m}}{(m-n) !} \leq \sum_{m=1}^{\infty} \frac{a_{m}}{m !} \leq \sum_{n=1}^{2 k-1} \sum_{m=n}^{\infty} \frac{(-1)^{n+1}}{n !} \frac{a_{m}}{(m-n) !} .
$$

Interchanging the order of summation shows that these inequalities are implied by

$$
\sum_{n=1}^{(2 k) \wedge m} \frac{(-1)^{n+1}}{n !} \frac{1}{(m-n) !} \leq \frac{1}{m !} \leq \sum_{n=1}^{(2 k-1) \wedge m} \frac{(-1)^{n+1}}{n !} \frac{1}{(m-n) !}, \quad m \in \mathbb{N},
$$

where $(2 k) \wedge m$ denotes the minimum of $2 k$ and $m$. Since the latter equalities are equivalent to the elementary inequalities

$$
\sum_{n=0}^{(2 k) \wedge m}(-1)^{n}\left(\begin{array}{l}
m \\
n
\end{array}\right) \geq 0 \geq \sum_{n=0}^{(2 k-1) \wedge m}(-1)^{n}\left(\begin{array}{c}
m \\
n
\end{array}\right)
$$

this concludes the proof of (1.7). Moreover, the first inequality in (4.3) is strict unless $m \leq 2 k$, so that the first inequality of (1.7) is an equality if and only if $a_{m}=0$ for all $m>$ $2 k$. By Theorem 1.3 and Proposition 4.1, this happens if and only if $D_{y_{1}, \ldots, y_{2 k+1}}^{2 k+1} f(\eta)=0$ almost surely for $\lambda^{2 k+1}$-a.e. $\left(y_{1}, \ldots, y_{2 k+1}\right)$.

Similarly, the second inequality in (4.3) is strict unless $m \leq 2 k-1$, so that the second inequality of (1.7) is an equality if and only if $a_{m}=0$ for all $m \leq 2 k-1$, which happens if and only if $D_{y_{1}, \ldots, y_{2 k}}^{2 k} f(\eta)=0$ almost surely for $\lambda^{2 k}$-a.e. $\left(y_{1}, \ldots, y_{2 k}\right)$.

\section{Covariance identities}

In this section we shall prove Theorem 1.5 but first we give some of its consequences. 
Theorem 5.1. Consider a Poisson process $\tilde{\eta}$ on $\tilde{\mathbb{Y}}:=\mathbb{R}_{+} \times \mathbb{Y}$ whose intensity measure is the product of Lebesgue measure on $[0,1]$ and $\lambda$. Assume that $\eta=\tilde{\eta}([0,1] \times \cdot)$, and for $s>0$ let $\tilde{\eta}_{s-}$ denote the restriction of $\tilde{\eta}$ to $[0, s) \times \mathbb{Y}$. Let $f, g \in L^{2}\left(\mathbb{P}_{\eta}\right)$. Then

$$
\mathbb{E} \iint_{0}^{1} \mathbb{E}\left[D_{y} f(\eta) \mid \tilde{\eta}_{s-}\right]^{2} d s \lambda(d y)<\infty
$$

and

$$
\operatorname{Cov}[f(\eta), g(\eta)]=\mathbb{E} \iint_{0}^{1} \mathbb{E}\left[D_{y} f(\eta) \mid \tilde{\eta}_{s-}\right] \mathbb{E}\left[D_{y} g(\eta) \mid \tilde{\eta}_{s-}\right] d s \lambda(d y)
$$

Proof: Apply Theorem 1.5 to $\tilde{\eta}$ with the relation $<$ on $\tilde{\mathbb{Y}}$ given by $(s, x)<(t, y)$ if and only if $s<t$.

Proof of Theorem 1.4: It is no loss of generality to assume that $\eta=\tilde{\eta}([0,1] \times \cdot)$, with $\tilde{\eta}$ as in Theorem 5.1. Then the result is a direct consequence of Theorem 5.1.

Remark 5.2. Again assuming without loss of generality that $\eta=\tilde{\eta}([0,1] \times \cdot)$, taking $g=f$ and applying the conditional Jensen inequality in (5.2) yields an alternative proof of the Poincaré inequality (1.8).

We proceed with proving Theorem 1.5. For the rest of this section we assume that $\eta$ is a Poisson process on $\mathbb{Y}=\mathbb{R}_{+} \times \mathbb{X}$ with an intensity measure satisfying (1.11). We need the following lemma.

Lemma 5.3. Let $f \in L^{2}\left(\mathbb{P}_{\eta}\right)$ and define $f_{y}(\mu):=\int D_{y} f\left(\mu_{y}+\nu\right) \Pi^{y}(d \nu)$, for $\mu \in \mathbf{N}$, $y \in \mathbb{Y}$. Then

$$
\mathbb{E} D_{\left(x_{1}, \ldots, x_{n}\right)}^{n} f_{y}(\eta)=\left(\prod_{i=1}^{n} \mathbf{1}\left\{x_{i}<y\right\}\right) \mathbb{E} D_{\left(x_{1}, \ldots, x_{n}, y\right)}^{n+1} f(\eta)
$$

holds for $\lambda^{n+1}$-a.e. $\left(x_{1}, \ldots, x_{n}, y\right)$.

Proof: For any $n \in \mathbb{N}$, and any $C \in \mathcal{Y}_{0}$, arguing as in the proof of Lemma 2.3 yields

$$
\int_{C^{n}} \mathbb{E}\left[\left|f\left(\eta+\sum_{i=1}^{n} \delta_{y_{i}}\right)\right|\right] \lambda^{n}\left(d\left(y_{1}, \ldots, y_{n}\right)\right)<\infty .
$$

Hence $\mathbb{E}\left[\left|f\left(\eta+\sum_{i=1}^{n} \delta_{y_{i}}\right)\right|\right]$ is finite for $\lambda^{n}$-almost all $\left(y_{1}, \ldots, y_{n}\right) \in \mathbb{Y}^{n}$. Thus for any $m \in \mathbb{N}_{0}$,

$$
\int\left|f\left(\mu_{y}+\delta_{y_{1}}+\ldots+\delta_{y_{m}}+\delta_{y}+\nu\right)\right| \Pi^{y}(d \nu)<\infty
$$

and

$$
\int\left|f\left(\mu_{y}+\delta_{y_{1}}+\ldots+\delta_{y_{m}}+\nu\right)\right| \Pi^{y}(d \nu)<\infty
$$


hold for $\mathbb{P}_{\eta^{-}}$a.e. $\mu$ and $\lambda^{m+1}$-a.e. $\left(y_{1}, \ldots, y_{m}, y\right)$, since integrating the left hand side of (5.4) over $\mu$ (with respect to the measure $\mathbb{P}_{\eta}$ ) yields $\mathbb{E}\left|f\left(\eta+\delta_{y}+\sum_{i=1}^{m} \delta_{y_{i}}\right)\right|$ and integrating the left hand side of (5.5) over $\mu$ yields $\mathbb{E}\left|f\left(\eta+\sum_{i=1}^{m} \delta_{y_{i}}\right)\right|$.

Let $x_{i} \in \mathbb{Y}$ for $1 \leq i \leq n$. First suppose for some $i$ that $x_{i}<y$ does not hold. Then since $f_{y}(\mu)$ depends on $\mu$ only through $\mu_{y}$, and since $\left(\mu+\delta_{x_{i}}\right)_{y}=\mu_{y}$, it follows that $D_{x_{i}} f_{y}(\mu)=0$ for any $\mu$. Since $D_{y_{1}, \ldots, y_{n}} f(\mu)$ is symmetric in $y_{1}, \ldots, y_{n}$ it follows that in this case (5.3) holds with both sides equal to zero.

Now suppose that $x_{i}<y$ for each $i$, and that (5.4) and (5.5) hold for every subset $\left\{y_{1}, \ldots, y_{m}\right\}$ of $\left\{x_{1}, \ldots, x_{n}\right\}$. Then by $(2.4)$

$$
\begin{aligned}
D_{x_{1}, \ldots, x_{n}}^{n} f_{y}(\mu) & =\sum_{J \subset\{1, \ldots, n\}}(-1)^{n-|J|} f_{y}\left(\mu+\sum_{i \in J} \delta_{x_{i}}\right) \\
& =\sum_{J \subset\{1, \ldots, n\}}(-1)^{n-|J|} \int D_{y} f\left(\left(\mu+\sum_{i \in J} \delta_{x_{i}}\right)_{y}+\nu\right) \Pi^{y}(d \nu) \\
& =\int D_{y, x_{1}, \ldots, x_{n}}^{n+1} f\left(\mu_{y}+\nu\right) \Pi^{y}(d \nu) .
\end{aligned}
$$

Integrating over $\mu$ (with respect to the measure $\mathbb{P}_{\eta}$ ) yields (5.3) for this case.

Proof of Theorem 1.5: Let us first assume that $f, g$ are bounded. Then $f_{y}$ and $g_{y}$ are trivially in $L^{2}\left(\mathbb{P}_{\eta}\right)$ for all $y \in \mathbb{Y}$ and we have by (1.12) that

$$
\mathbb{E} \int \mathbb{E}\left[D_{y} f(\eta) \mid \eta_{y}\right] \mathbb{E}\left[D_{y} g(\eta) \mid \eta_{y}\right] \lambda(d y)=\int \mathbb{E}\left[f_{y}(\eta) g_{y}(\eta)\right] \lambda(d y),
$$

where the use of Fubini's theorem will be justified below. By Theorem 1.1 and Lemma 5.3 this equals

$$
\int \sum_{n=0}^{\infty} \frac{1}{n !}\left\langle T_{n} f_{y}, T_{n} g_{y}\right\rangle_{n} \lambda(d y)=\sum_{n=0}^{\infty} \frac{1}{n !}\left\langle\mathbf{1}_{J_{n+1}} T_{n+1} f, T_{n+1} g\right\rangle_{n+1},
$$

where $J_{n+1}:=\left\{\left(y_{1}, \ldots, y_{n+1}\right): y_{1}<y_{n+1}, \ldots, y_{n}<y_{n+1}\right\}$ and where the interchange of summation and integration will be justified below. By (1.11) the measure $\lambda^{n+1}$ is concentrated on $\cup_{\pi \in \Sigma_{n+1}}\left\{\left(y_{1}, \ldots, y_{n+1}\right): y_{\pi(1)}<\ldots<y_{\pi(n+1)}\right\}$ where the union is over all permutations. Using the symmetry of $T_{n+1} f$ and $T_{n+1} g$ this gives

$$
\frac{1}{n !}\left\langle\mathbf{1}_{J_{n+1}} T_{n+1} f, T_{n+1} g\right\rangle_{n+1}=\frac{1}{(n+1) !}\left\langle T_{n+1} f, T_{n+1} g\right\rangle_{n+1} .
$$

Using Theorem 1.1 again, yields the asserted identity (1.14). Repeating the above calculation with $f=g$ shows that (1.13) holds. This also justifies our use of Fubini's theorem.

Finally we consider the case of general $f, g \in L^{2}\left(\mathbb{P}_{\eta}\right)$. The previous arguments carry through once we have shown that (1.13) holds for $f \in L^{2}\left(\mathbb{P}_{\eta}\right)$. Let $f^{k}, k \in \mathbb{N}$, be a sequence of bounded measurable functions on $\mathbf{N}(\mathbb{Y})$ such $\mathbb{E}\left(f(\eta)-f^{k}(\eta)\right)^{2} \rightarrow 0$ as $k \rightarrow \infty$. We have just proved that

$$
\operatorname{Var}\left[f^{k}(\eta)-f^{l}(\eta)\right]=\mathbb{E} \int\left(\mathbb{E}\left[D_{y} f^{k}(\eta) \mid \eta_{y}\right]-\mathbb{E}\left[D_{y} f^{l}(\eta) \mid \eta_{y}\right]\right)^{2} \lambda(d y), \quad k, l \in \mathbf{N} .
$$


Since $L^{2}(\mathbb{P} \otimes \lambda)$ is complete, there is an $h \in L^{2}(\mathbb{P} \otimes \lambda)$ satisfying

$$
\lim _{k \rightarrow \infty} \mathbb{E} \int\left(h(y)-\mathbb{E}\left[D_{y} f^{k}(\eta) \mid \eta_{y}\right]\right)^{2} \lambda(d y)=0 .
$$

On the other hand it follows from Lemma 2.3 that for any $C \in \mathcal{Y}_{0}$

$$
\begin{aligned}
\int_{C} \mathbb{E} \mid \mathbb{E}\left[D_{y} f^{k}(\eta) \mid \eta_{y}\right] & -\mathbb{E}\left[D_{y} f(\eta) \mid \eta_{y}\right] \mid \lambda(d y) \\
& \leq \int_{C} \mathbb{E}\left|D_{y} f^{k}(\eta)-D_{y} f(\eta)\right| \lambda(d y) \rightarrow 0
\end{aligned}
$$

as $k \rightarrow \infty$. Comparing this with (5.7) shows that $h(\omega, y)=\mathbb{E}\left[D_{y} f \mid \eta_{y}\right](\omega)$ for $\mathbb{P} \otimes \lambda$-a.e. $(\omega, y) \in \Omega \times C$ and hence also for $\mathbb{P} \otimes \lambda$-a.e. $(\omega, y) \in \Omega \times \mathbb{Y}$. Therefore the fact that $h \in L^{2}\left(\mathbb{P}_{\eta} \otimes \lambda\right)$ implies (1.13).

\section{$6 \quad$ Infinitely divisible random measures}

In this section we consider an infinitely divisible random measure $\xi$ on a complete separable metric space $\mathbb{X}$ equipped with the Borel $\sigma$-field $\mathcal{X}$, see $[24,6]$ and $[20]$ for the the special case of a locally compact phase space $\mathbb{X}$. The distribution of such a random measure can be most conveniently described by its Laplace functional as follows. Let $\mathbf{M}$ denote the space of all locally finite measure on $\mathbb{X}$, that is, the set of all measures that are finite on metrically bounded sets. We equip $\mathbf{M}$ with the smallest $\sigma$-field of subsets of $\mathbf{M}$ such that the mappings $\mu \mapsto \mu(B)$ are measurable for all $B \in \mathcal{X}$. Let $v: \mathbb{X} \rightarrow \mathbb{R}_{+}$be measurable. Then

$$
\mathbb{E} \exp \left[-\int v d \xi\right]=\exp \left[-\int v d \alpha-\int\left(1-e^{-\int v d \mu}\right) \mathbb{Q}(d \mu)\right]
$$

where $\alpha \in \mathbf{M}$ and the $K L M$ (or Lévy) measure $\mathbb{Q}$ is a $\sigma$-finite measure on $\mathbf{M}$ having $\mathbb{Q}(\{0\})=0$ (here 0 denotes the zero measure) and

$$
\int(1-\exp [-\mu(B)]) \mathbb{Q}(d \mu)<\infty, \quad B \in \mathcal{X}_{0}
$$

Here $\mathcal{X}_{0} \subset \mathcal{X}$ denotes the ring of (metrically) bounded Borel subsets of $\mathbb{X}$.

Proposition 6.1. For any $f \in L^{2}\left(\mathbb{P}_{\xi}\right)$,

$$
\operatorname{Var}[f(\xi)] \leq \mathbb{E} \int(f(\xi+\mu)-f(\xi))^{2} \mathbb{Q}(d \mu)
$$

Proof: Let $C_{n}, n \in \mathbb{N}$, be a sequence of closed balls with fixed centre and radius $n$. We define a measurable mapping $H: \mathbf{N}(\mathbf{M}) \rightarrow \mathbf{M}$ as follows. Let $\nu \in \mathbf{N}(\mathbf{M})$ and define

$$
H(\nu)(B):=\alpha(B)+\int \mu(B) \nu(d \mu), \quad B \in \mathcal{X}
$$


whenever the right hand side is finite for all $B=C_{n}, n \in \mathbb{N}$. Otherwise we define $H(\nu):=0$. Now let $\eta$ be a Poisson process on $\mathbf{M}$ with intensity measure $\mathbb{Q}$. It is wellknown $[20,24,6]$ that $\xi$ and $H(\eta)$ have the same distribution. Because of this Poisson cluster representation of $\xi$ we can assume $\xi=H(\eta)$ without restricting generality. Now we apply Theorem 1.2 to the function $f \circ H$. Since $H\left(\nu+\delta_{\mu}\right)=H(\nu)+\mu$ for all $\nu \in \mathbf{N}(\mathbf{M})$ and all $\mu \in \mathbf{M}$ we obtain the assertion.

In case $\alpha=0$ and $\mathbb{Q}=\int \mathbf{1}\left\{\delta_{x} \in \cdot\right\} \lambda^{\prime}(d x)$ for some $\lambda^{\prime} \in \mathbf{M}$ the random measure $\xi$ is a Poisson process with intensity measure $\lambda^{\prime}$. Then the inequality (6.3) simplifies to the Poincaré inequality (1.8).

By means of the Poisson cluster representation of $\xi$ we could also rewrite the other results of this paper. We restrict ourselves to the following version of the Harris-FKG inequality. We call a measurable function $f: \mathbf{M} \rightarrow \mathbb{R}$ increasing almost everywhere if $f(\xi+\mu) \geq f(\xi) \mathbb{P}$-a.s. and for $\mathbb{Q}$-a.e. $\mu$. The next proposition follows from Theorem 1.4.

Proposition 6.2. Assume that $f, g \in L^{2}\left(\mathbb{P}_{\xi}\right)$ are increasing almost everywhere. Then

$$
\mathbb{E}[f(\xi) g(\xi)] \geq(\mathbb{E} f(\xi))(\mathbb{E} g(\xi)) .
$$

\section{$7 \quad$ The case of finite $\mathbb{Y}$}

For this section only, we assume $\mathbb{Y}$ is a finite set $\{1, \ldots, k\}$ and $\mathcal{Y}$ is the power set of $\mathbb{Y}$. In this case let us write $\lambda_{i}$ for $\lambda(\{i\})$ (assumed finite) and $\eta_{i}$ for $\eta(\{i\})$. Then $\eta_{1}, \ldots, \eta_{k}$ are independent Poisson variables. Given $n \in \mathbb{N}$ and $\mathbf{y}=\left(y_{1}, \ldots, y_{n}\right) \in \mathbb{Y}^{n}$, let $\mathbf{m}(\mathbf{y})=\left(m_{1}(\mathbf{y}), \ldots, m_{k}(\mathbf{y})\right)$ be given by

$$
m_{j}(\mathbf{y}):=\sum_{i=1}^{n} \mathbf{1}\left\{y_{i}=j\right\}
$$

so that $\sum_{j=1}^{k} m_{j}(\mathbf{y})=n$. Then for any $h: \mathbb{Y}^{n} \rightarrow \mathbb{R}$, by (3.3) and linearity,

$$
I_{n}(h)=\sum_{\mathbf{y} \in \mathbb{Y}^{n}} h(\mathbf{y}) \prod_{i=1}^{k}\left(\lambda_{i}^{m_{i}} C_{m_{i}}\left(\lambda_{i} ; \eta_{i}\right)\right) .
$$

Now consider $f: \mathbb{N}_{0}^{k} \rightarrow \mathbb{R}$ with $\mathbb{E} f\left(\eta_{1}, \ldots, \eta_{k}\right)^{2}<\infty$. Then $T_{n} f\left(y_{1}, \ldots, y_{n}\right)$ depends on $\mathbf{y}$ only through $\mathbf{m}:=\mathbf{m}(\mathbf{y})$. Writing $m_{i}$ for $m_{i}(\mathbf{y})$, and using (2.4), we define

$$
a_{\mathbf{m}}:=T_{n} f(\mathbf{y})=\sum_{i_{1}=0}^{m_{1}} \ldots \sum_{i_{k}=0}^{m_{k}}(-1)^{n-\sum_{j=1}^{k} i_{j}}\left(\prod_{j=1}^{k}\left(\begin{array}{c}
m_{j} \\
i_{j}
\end{array}\right)\right) \mathbb{E}\left[f\left(\eta_{1}+i_{1}, \ldots, \eta_{k}+i_{k}\right)\right] .
$$

Then by (7.1),

$$
I_{n}\left(T_{n} f\right)=\sum_{\mathbf{m}: m_{1}+\ldots+m_{k}=n}\left(\frac{n !}{m_{1} ! \cdots m_{k} !}\right) a_{\mathbf{m}} \prod_{i=1}^{k}\left(\lambda_{i}^{m_{i}} C_{m_{i}}\left(\lambda_{i} ; \eta_{i}\right)\right)
$$


and therefore by Theorem 1.3,

$$
f\left(\eta_{1}, \ldots, \eta_{k}\right)=\mathbb{E}\left[f\left(\eta_{1}, \ldots, \eta_{k}\right)\right]+\sum_{\mathbf{m}} a_{\mathbf{m}} \prod_{i=1}^{k}\left(\lambda_{i}^{m_{i}} C_{m_{i}}\left(\lambda_{i} ; \eta_{i}\right) / m_{i} !\right)
$$

where the sum is over all $\mathbf{m}=\left(m_{1}, \ldots, m_{k}\right) \in \mathbb{N}_{0}^{k}$ except for $(0, \ldots, 0)$, and the convergence is in $L^{2}(\mathbb{P})$.

This identifies the coefficients in the Charlier polynomial expansion of $f$ in terms of the expected repeated differences $a_{\mathbf{m}}$. Note that $\mathbb{E}\left[C_{m}\left(\lambda_{i} ; \eta_{i}\right)^{2}\right]=m ! \lambda_{i}^{-m}$ (to see this, use (7.1) and (3.5) in the case with $k=1$ and $h=g=1$ ). Hence by taking inner products with $\prod_{i=1}^{k} C_{m_{i}}\left(\lambda_{i} ; \eta_{i}\right)$ in $(7.2)$ and using orthogonality of the Charlier polynomials, we obtain

$$
a_{\mathbf{m}}=\mathbb{E}\left[f\left(\eta_{1}, \ldots, \eta_{k}\right) \prod_{i=1}^{k} C_{m_{i}}\left(\lambda_{i} ; \eta_{i}\right)\right] .
$$

In the case $k=1$ this has previously been obtained as Lemma 9.1.4 of [2].

Acknowledgements: We wish to thank Yuri Kabanov for giving several insightful comments on an early draft of this paper and Liming Wu for sending us his unpublished work [42]. Thanks are also due to Matthias Reitzner, who drew our attention to the Poincaré inequality for general Poisson processes.

\section{References}

[1] Ané, C. and Ledoux, M. (2000). On logarithmic Sobolev inequalities for continuous time random walks on graphs. Probab. Theory Related Fields 116, 573-602.

[2] Barbour, A. D., Holst, L. and Janson, S. (1992). Poisson Approximation. Clarendon Press, Oxford.

[3] Blaszczyszyn, B. (1995). Factorial-moment expansion for stochastic systems. Stoch. Proc. Appl. 56, 321-335.

[4] Bollobás, B. and Riordan, O. (2006). Percolation. Cambridge University Press, New York,

[5] Chen, L. (1985). Poincaré-type inequalities via stochastic integrals. Z. Wahrscheinlichkeitstheorie verw. Gebiete 69, 251-277.

[6] Daley, D.J. and Vere-Jones, D. (2008). An Introduction to the Theory of Point Processes, Volume II. 2nd ed., Springer, New York.

[7] Dellacherie, C. and Meyer, P.A. (1978). Probabilities and potential. Mathematics Studies, Volume 29, Hermann, Paris. North-Holland Publishing Company, Amsterdam and New York.

[8] Efron, B. and Stein, C. (1981). The jackknife estimate of variance. Ann. Statist. 9, $586-596$. 
[9] Georgii, H. and Küneth, T. (1997). Stochastic order of point processes. J. Appl. Prob. 34, 868-881.

[10] Heveling, M. and Reitzner, M. (2009). Poisson-Voronoi approximation Ann. Appl. Probab. 19, 719-736.

[11] Hitsuda, M. (1972). Formula for Brownian partial derivatives. Proceedings of the 2nd Japan-USSR Symposium on Probability Theory. 111-114.

[12] Houdré, C., and Perez-Abreu, V. (1995). Covariance identities and inequalities for functionals on Wiener space and Poisson space. Ann. Probab. 23, 400-419.

[13] Houdré, C. (2002). Remarks on deviation inequalities for functions of infinitely divisible random vectors. Ann. Probab. 30, 1223-1237.

[14] Houdré, C. and Privault, N. (2002). Concentration and deviation inequalities in infinite dimensions via covariance representations. Bernoulli 8, 697-720.

[15] Itô, K. (1951). Multiple Wiener integral. J. Math. Soc. Japan 3, 157-169.

[16] Itô, K. (1956). Spectral type of the shift transformation of differential processes with stationary increments. Trans. Amer. Math. Soc. 81, 253-263.

[17] Ito, Y. (1988). Generalized Poisson functionals. Probab. Th. Rel. Fields 77, 1-28.

[18] Kabanov, Y.M. (1975). On extended stochastic integrals. Theory Probab. Appl. 20, 710-722.

[19] Kabanov, Y.M. and Skorokhod, A.V. (1975). Extended stochastic integrals. Proceedings of the School-Seminar on the Theory of Random Processes. Druskininkai, November 25-30, 1974. Part I. Vilnius, (Russian).

[20] Kallenberg, O. (1983). Random Measures. Akademie-Verlag Berlin and Academic Press, London.

[21] Kallenberg, O. (2002). Foundations of Modern Probability. Second Edition, Springer, New York.

[22] Liebscher, V. (1994): On the isomorphism of Poisson space and symmetric Fock Space. In L. Accardi, editor, Quantum Probability $\&$ Related Topics IX, pp. 295-300, World Scientific, Singapore, .

[23] Løkka, A. (2005). Martingale representation of functionals of Lévy processes. Stochastic Analysis and Applications 22, 867-892.

[24] Matthes, K., Kerstan, J. and Mecke, J. (1978). Infinitely Divisible Point Processes. Wiley, Chichester.

[25] Mecke, J. (1967). Stationäre zufällige Maße auf lokalkompakten Abelschen Gruppen. Z. Wahrsch. verw. Gebiete 9, 36-58.

[26] Meester, R. and Roy, R. (1996). Continuum Percolation, Cambridge Univ. Press. 
[27] Meyer, P.A. (1995). Quantum Probability for Probabilists 2nd ed., Springer, Berlin.

[28] Møller, J. and Zuyev, S. (1996). Gamma-type results and other related properties of Poisson processes. Adv. Appl. Prob. 28, 662-673.

[29] Molchanov, I. and Zuyev, S (2000). Variational analysis of functionals of Poisson processes. Math. Operat. Res. 25, 485-508.

[30] Nualart, D. and Vives, J. (1990). Anticipative calculus for the Poisson process based on the Fock space. In: Séminaire de Probabilités XXIV, Lecture Notes in Math., 1426, 154-165.

[31] Ogura, H. (1972). Orthogonal functionals of the Poisson processes. Trans IEEE Inf. Theory 18, 473-481.

[32] Peccati, G., Solé, J. L., Taqqu, M.S. and Utzet, F. (2009). Stein's method and normal approximation of Poisson functionals. Ann. Probab., to appear.

[33] Penrose, M. (2003). Random geometric graphs. Oxford University Press, Oxford.

[34] Penrose, M. D. and Sudbury, A. (2005). Exact and approximate results for deposition and annihilation processes on graphs. Ann. Appl. Probab. 15 853-889.

[35] Penrose, M.D. and Wade, A.R. (2008). Multivariate normal approximation in geometric probability. Journal of Statistical Theory and Practice 2, 293-326.

[36] Picard, J. (1996). Formules de dualité sur lespace de Poisson. Ann. Inst. H. Poincaré Probab. Statist. 32, 509-548.

[37] Picard, J. (1996). On the existence of smooth densities for jump processes. Probab. Theory Related Fields 105, 481-511.

[38] Privault, N. (2001). Extended covariance identities and inequalities. Statistics \& Probability Letters 55, 247-255.

[39] Surgailis, D. (1984) On multiple Poisson stochastic integrals and associated Markov semigroups. Probab. Math. Statist. 3, 217-239.

[40] Skorohod, A. V. (1975). On a generalization of a stochastic integral. Theory Probab. Appl. 20, 219-233.

[41] Wiener, N. (1938). The homogeneous chaos. Am J. Math. 60, 897-936.

[42] Wu, L. (1998). $L^{1}$ and modified logarithmic Sobolev inequalities and deviation inequalities for Poisson point processes. Preprint.

[43] Wu, L. (2000). A new modified logarithmic Sobolev inequality for Poisson point processes and several applications. Probab. Theory Related Fields 118, 427-438. 